\title{
Thermodynamic correction of particle concentrations measured by underwing probes on fast-flying aircraft
}

\author{
Ralf Weigel $^{1}$, Peter Spichtinger ${ }^{1}$, Christoph Mahnke ${ }^{1,2}$, Marcus Klingebiel ${ }^{2, a}$, Armin Afchine ${ }^{3}$, Andreas Petzold ${ }^{4}$, \\ Martina Krämer ${ }^{3}$, Anja Costa ${ }^{3}$, Sergej Molleker ${ }^{2}$, Philipp Reutter $^{1}$, Miklós Szakáll ${ }^{1}$, Max Port ${ }^{2}$, Lucas Grulich ${ }^{5}$, \\ Tina Jurkat ${ }^{6}$, Andreas Minikin ${ }^{6, b}$, and Stephan Borrmann ${ }^{1,2}$ \\ ${ }^{1}$ Institut für Physik der Atmosphäre, Johannes Gutenberg University, Mainz, Germany \\ ${ }^{2}$ Particle Chemistry Department, Max Planck Institute for Chemistry, Mainz, Germany \\ ${ }^{3}$ Institut für Energie- und Klimaforschung (IEK-7), Forschungszentrum Jülich, Jülich, Germany \\ ${ }^{4}$ Institut für Energie- und Klimaforschung (IEK-8), Forschungszentrum Jülich, Jülich, Germany \\ ${ }^{5}$ Institute of Computer Science, Johannes Gutenberg University, Mainz, Germany \\ ${ }^{6}$ Institut für Physik der Atmosphäre, Deutsches Zentrum für Luft- und Raumfahrt (DLR), Oberpfaffenhofen, Germany \\ ${ }^{a}$ now at: Max Planck Institute for Meteorology, Hamburg, Germany \\ ${ }^{b}$ now at: Abteilung Flugexperimente (FX), Deutsches Zentrum für Luft- und Raumfahrt (DLR), Oberpfaffenhofen, Germany
}

Correspondence to: Ralf Weigel (weigelr@uni-mainz.de)

Received: 2 December 2015 - Published in Atmos. Meas. Tech. Discuss.: 18 December 2015

Revised: 26 September 2016 - Accepted: 27 September 2016 - Published: 20 October 2016

\begin{abstract}
Particle concentration measurements with underwing probes on aircraft are impacted by air compression upstream of the instrument body as a function of flight velocity. In particular, for fast-flying aircraft the necessity arises to account for compression of the air sample volume. Hence, a correction procedure is needed to invert measured particle number concentrations to ambient conditions that is commonly applicable to different instruments to gain comparable results. In the compression region where the detection of particles occurs (i.e. under factual measurement conditions), pressure and temperature of the air sample are increased compared to ambient (undisturbed) conditions in certain distance away from the aircraft. Conventional procedures for scaling the measured number densities to ambient conditions presume that the air volume probed per time interval is determined by the aircraft speed (true air speed, TAS). However, particle imaging instruments equipped with pitot tubes measuring the probe air speed (PAS) of each underwing probe reveal PAS values systematically below those of the TAS. We conclude that the deviation between PAS and TAS is mainly caused by the compression of the probed air sample. From measurements during two missions in 2014 with the German Gulfstream G-550 (HALO - High Altitude LOng range) research aircraft we develop a procedure to correct the mea-
\end{abstract}

sured particle concentration to ambient conditions using a thermodynamic approach. With the provided equation, the corresponding concentration correction factor $\xi$ is applicable to the high-frequency measurements of the underwing probes, each of which is equipped with its own air speed sensor (e.g. a pitot tube). $\xi$ values of 1 to 0.85 are calculated for air speeds (i.e. TAS) between 60 and $250 \mathrm{~m} \mathrm{~s}^{-1}$. For different instruments at individual wing position the calculated $\xi$ values exhibit strong consistency, which allows for a parameterisation of $\xi$ as a function of TAS for the current $H A L O$ underwing probe configuration. The ability of cloud particles to adopt changes of air speed between ambient and measurement conditions depends on the cloud particles' inertia as a function of particle size (diameter $D_{\mathrm{p}}$ ). The suggested inertia correction factor $\mu\left(D_{\mathrm{p}}\right)$ for liquid cloud drops ranges between 1 (for $D_{\mathrm{p}}<70 \mu \mathrm{m}$ ) and 0.8 (for $100 \mu \mathrm{m}<D_{\mathrm{p}}<225 \mu \mathrm{m}$ ) but it needs to be applied carefully with respect to the particles' phase and nature. The correction of measured concentration by both factors, $\xi$ and $\mu\left(D_{\mathrm{p}}\right)$, yields higher ambient particle concentration by about $10-25 \%$ compared to conventional procedures - an improvement which can be considered as significant for many research applications. The calculated $\xi$ values are specifically related to the considered $H A L O$ underwing probe arrangement and may differ for other air- 
craft. Moreover, suggested corrections may not cover all impacts originating from high flight velocities and from interferences between the instruments and e.g. the aircraft wings and/or fuselage. Consequently, it is important that PAS (as a function of TAS) is individually measured by each probe deployed underneath the wings of a fast-flying aircraft.

\section{Introduction}

Clouds constitute one of the most important regulators of the Earth's energy balance. The radiation net effect of various cloud types it is not ultimately known yet. The albedo effect and the greenhouse effect of clouds are driven by the cloud element's microphysical properties (e.g. the particles' number, size and shape). In a first-order estimate the cloud particle size is mostly determined by the cloud particle number concentration, since the available water vapour for condensation is distributed via diffusion over the number of particles present within a cloud. Cloud particle number concentrations are highly variable (e.g. Krämer et al., 2009), typically ranging from a few thousandths up to hundreds of particles per cubic centimetre, since specific mechanisms of cloud formation are determined by local dynamics (e.g. Spichtinger and Gierens, 2009; Kärcher and Lohmann, 2002).

Airborne in situ investigations related to the microphysical properties of cloud particles, ice crystals and hydrometeors are essential for answering many scientific questions and therefore measurement methods by means of underwing probes are widely used (cf. Baumgardner et al., 2011; Wendisch and Brenguier, 2013). Airborne in situ measurements of cloud elements are generally influenced by aerodynamic conditions at the instrument's individual mounting position, i.e. due to specific flow fields around the aircraft's fuselage and wings (Drummond and MacPherson, 1985; Norment, 1988). Local fluctuations of the air density may occur in the vicinity of measurement instruments and their sensing volumes (MacPherson and Baumgardner, 1988), which can affect typical measurements like particle number concentrations and subsequently derived distributions of surface areas or volumes. Consequently, if possible, the thermodynamic conditions during particle detection need to be considered for gaining accurate and comparable results.

Two scientific missions were carried out in 2014 with the German Gulfstream G-550 (HALO - High Altitude LOng range), the sister ship of the US research aircraft HIAPER (High-Performance Instrumented Airborne Platform for Environmental Research) (Laursen et al., 2006): (1) MLCIRRUS, from 24 March to 30 April with a total of $\sim 71$ measurement flight hours at midlatitudes over Central Europe (Voigt et al., 2016), and (2) ACRIDICON-CHUVA, during September, with overall $\sim 96$ local mission flight hours in tropical regions, over the Amazonian basin, Brazil. During both missions, several independent underwing probes were deployed (e.g. the Cloud Combination Probe - CCP; the Small Ice Detector - SID3; the Cloud, Aerosol and Precipitation Spectrometer - CAPS; the Coud and Aerosol Spectrometer - CAS; the Precipitation Imaging Probe - PIP; and the Particle Habit Imaging and Polar Scattering Probe - PHIPS) for studies concerning cloud particle microphysical properties at relative high flight velocities reached by $H A L O$ (up to Mach 0.75). Thus, the impacts on the air flow conditions towards underwing probes, previously considered numerically for flight velocities between 50 and $130 \mathrm{~m} \mathrm{~s}^{-1}$ (Norment and Quealy, 1988) and empirically for up to $100 \mathrm{~m} \mathrm{~s}^{-1}$ (MacPherson and Baumgardner, 1988), need to be reassessed for the air compression accompanied with high flight velocities.

The diagram in Fig. 1 shows an aircraft fuselage under flight conditions when passing a field of enhanced particle concentration, e.g. a cloud. By means of avionic (meteorological) sensors in the air data boom (cf. Fig. 1b; also referred to as nose boom) the ambient static air pressure $\left(p_{1}\right)$ and temperature $\left(T_{1}\right)$ are almost undisturbedly measured. The dynamic pressure proportion provided by the aircraft avionic sensors is transferable into the true air speed (TAS) according to Bernoulli's law and describes the aircraft speed relative to the current motion of air.

The underwing instrument probes are contained inside Particle Measuring Systems (PMS) standard canisters (with outer diameter of $\sim 177 \mathrm{~mm}$ ) which, in the HALO configuration, are pairwise mounted at an underwing pylon such that the instrument is placed $360 \mathrm{~mm}( \pm 30 \mathrm{~mm})$ underneath the aircraft wings. The instruments' detection volume is positioned $\sim 100 \mathrm{~mm}$ upstream of the wing's leading edge. This particular underwing positioning of the cloud probes partly results from pioneer work done in the past by comprehensive investigations concerning the impact of the air flow in the vicinity of an aircraft's wing on cloud particle trajectories (King, 1984; King, 1986a, b; Norment, 1988). Furthermore, detailed computational fluid dynamics (CFD) simulations were performed to optimise the particular underwing probe positioning on $H A L O$, as well as for other purposes. However, the publication of any result of these doubtlessly valuable studies, for which detailed aircraft geometry data of the G550 HALO were used, is not permitted as a consequence of a proprietary information agreement with the aircraft's manufacturer (K. Witte, German Aerospace Centre, personal communications, 2016).

The instruments' probe head has a quasi-aerodynamic shape (individual probe head designs of three different instruments are shown in Fig. 6). The individual probe head of the respective instrument is additionally characterised by extension arms that include the detection laser optics or other annexes such as the CAS winglet. Although the probe heads are generally of streamlined shape, the moving probe constitutes a flow resistance during flight due to the instrument body's cross-sectional area perpendicular to the direction of the air flow (cf. Fig. 1a). Thus, a compression region forms in a distance of $0.3-0.5 \mathrm{~m}$ upstream of the probe 


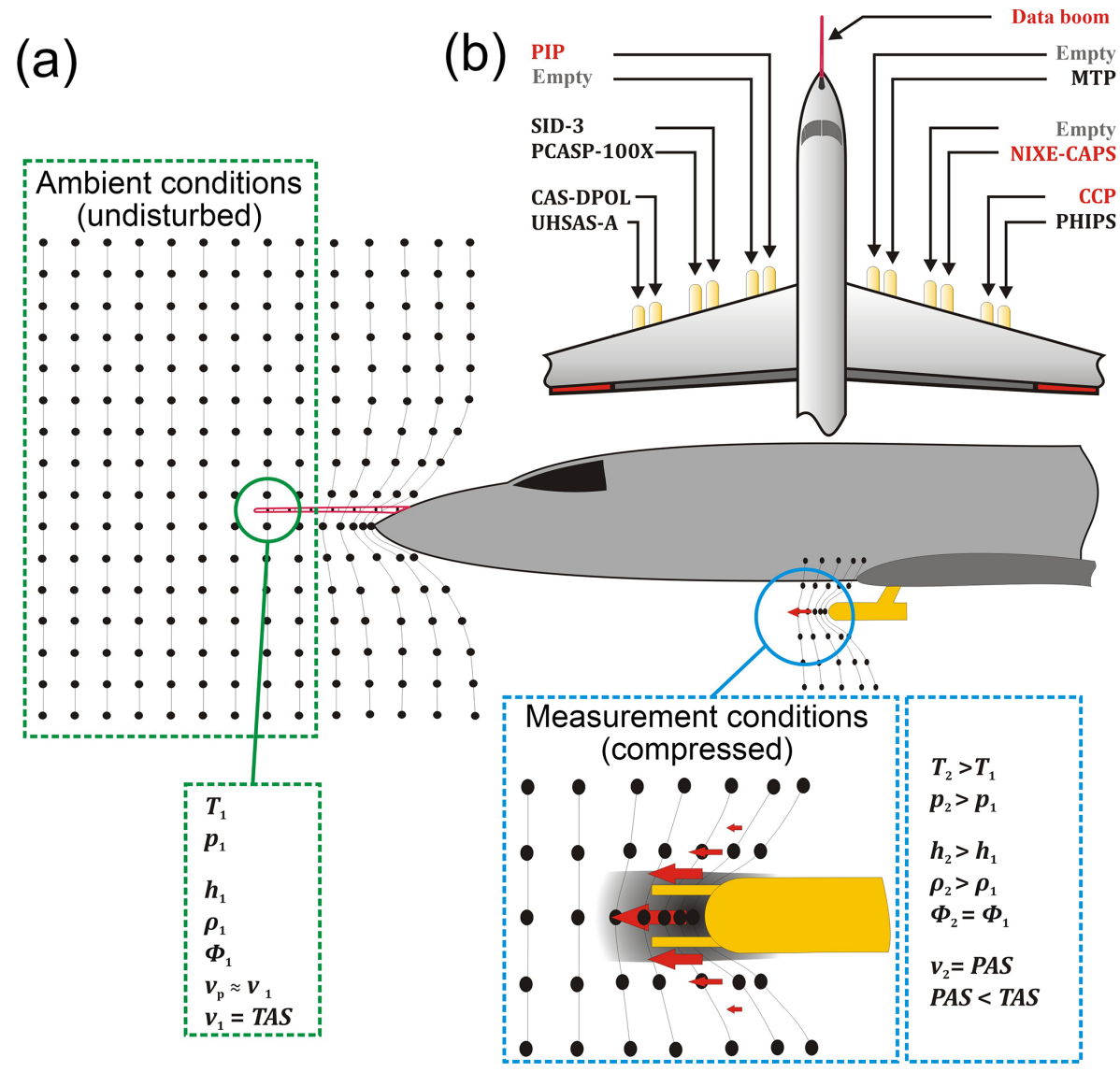

Figure 1. Illustration of the aircraft geometry, different types of probes and their underwing position and air compression effects. (a) The moving aircraft induces an increase of particle concentration in particular upstream of the underwing probes (grey-shaded area). Parameters used for deriving a thermodynamic correction are listed for ambient (undisturbed) conditions (green box) and for measurement conditions (blue box). Note that specified velocities refer to the moving aircraft or instrument relative to the air or the particles. (b) The top-view diagram of the aircraft indicates the probe's mounting position during the ML-CIRRUS and the ACRIDICON-CHUVA field missions. Data originating from the probes indicated in red are used for this study. (Instrument name acronyms are specified in the text.)

head (Wendisch and Brenguier, 2013) and the strength of compression is a function of aircraft speed. Further flowdynamical influences resulting from the proximity of the instruments to the wings or the fuselage of an aircraft may contribute to the modifications on the flow conditions (Drummond and MacPherson, 1985; MacPherson and Baumgardner, 1988). Primarily, the air compression due to the moving instrument body decelerates the air speed measurable at the probe, as the probe air speed (PAS) (DMT, 2009), whereby the rate of deceleration is a function of TAS. Furthermore, the compression of air results in the densification of the airborne particles at the point of measurement, i.e. well inside the compression region. This means that the particle concentrations measured under compressed conditions need to be scaled to ambient condition.

We aim to formulate an expression that is based on a thermodynamic approach to provide a correction factor for inverting measured particle number concentrations to ambient conditions. The variables contained in the corrective expres- sion should be available from meteorological data that are generally measured during research flights. Further variables of the measurement conditions should be available from the instrument itself, provided that it is equipped with a pitot tube. The effective correction may vary for the different instruments, the aircraft type and the position of the probe relative to the aircraft wings and/or fuselage. However, if the instrument is not equipped with a pitot tube, or the pitot tube is inoperative, the air speed at the point of measurement is unknown. In such a case the herein provided parameterisation of the compression correction serves as a guideline for adopting the TAS from the aircraft data after adjustments. In the following, the application of both the derived thermodynamic correction and the unadjusted aircraft TAS on a data set of atmospheric measurements illustrates the sensitivity of the results to the employed procedure. Furthermore, we show that the thermodynamic correction is relatively insensitive to the instrument position with respect to the aircraft fuselage, and the correlations of instrument-specific correction factors 
demonstrate robustness and consistency of the suggested approach.

\section{Method}

In this section, we describe a new method for determining the number concentration of particles in a given air volume from measured quantities and from basic thermodynamics. We will particularly emphasise the difference between our approach and the conventionally used methods, which focus exclusively on geometrical considerations but neglect effects of air compression.

For the following examination some definitions need to be particularly introduced: all velocities that are specified as air speeds $\left(v_{1}, v_{2}\right.$, TAS, PAS $)$ and the velocities of particles $\left(v_{\mathrm{p}}\right)$ refer to the moving aircraft or instruments relative to the air as the reference system. Measurement conditions are those under which the measurement occurs in the detection region that is impacted by compression. Ambient (undisturbed) conditions relate to the initial state far away from the aircraft.

\subsection{Ambient versus measured particle number densities}

The measured number concentration $N_{\text {meas }}$ (in units of number per air volume) detected with underwing probes that have a free stream detection volume $V_{\text {meas }}$ is defined as

$N_{\text {meas }}=n \cdot \frac{1}{A_{\mathrm{s}} \cdot v_{\mathrm{p}}} \cdot \frac{1}{\Delta t}=\frac{n}{V_{\text {meas }}}$.

Here, $n$ denotes the number of particles detected during the time interval $\Delta t$ (in seconds), and $v_{\mathrm{p}}$ denotes the velocity (in $\mathrm{m} \mathrm{s}^{-1}$ ) of particles penetrating the sample area $A_{\mathrm{s}}$ (in square metres).

With PAS $=v_{2}$ the detection volume is defined as

$V_{\text {meas }}=A_{\mathrm{s}} \cdot v_{2} \cdot \Delta t$

The ambient particle number concentration in the undisturbed ambient air is given as

$N_{\mathrm{amb}}=\frac{n_{\mathrm{amb}}}{V_{1}}$,

with the number of particles $n_{\mathrm{amb}}$ and the ambient air volume $V_{1}$ (in cubic metres).

Due to the compression of air upstream of the instruments, the ambient volume $V_{1}$ converts into the volume $V_{2}$. Under the preliminary assumptions that

1. the particle number per mass $M$ of the air sample is not affected by compression (i.e. remains constant and thus $\left.\frac{n_{\mathrm{amb}}}{M}=\frac{n_{\mathrm{meas}}}{M}\right)$ and

2. the particles' inertia is negligible for given streamlines and the ideal gas law $\left(p \cdot V=M \cdot R_{\mathrm{S}} \cdot T\right.$; with $\left.[p]=\mathrm{kg} \mathrm{ms}^{-2}, \quad[V]=\mathrm{m}^{3}, \quad[M]=\mathrm{kg}, \quad[T]=\mathrm{K}\right) \quad$ applies, where $R_{\mathrm{S}}$ denotes the specific gas constant (in $\mathrm{J} \mathrm{kg}^{-1} \mathrm{~K}^{-1}$; while $\mathrm{J}=\mathrm{kg} \mathrm{m}^{2} \mathrm{~s}^{-2}$ ),

we end up with the following equation:

$\frac{n}{M}=$ const. $\Rightarrow$ amb $\frac{R_{\mathrm{s}} \cdot T_{1}}{p_{1} \cdot V_{1}}=n_{\text {meas }} \frac{R_{\mathrm{s}} \cdot T_{2}}{p_{2} \cdot V_{2}}$.

Then we can derive the expression for determining the ambient particle number concentration:

$N_{\mathrm{amb}} \cdot R_{\mathrm{S}} \cdot \frac{T_{1}}{p_{1}}=N_{\text {meas }} \cdot R_{\mathrm{S}} \cdot \frac{T_{2}}{p_{2}} \stackrel{v_{\mathrm{p}}=v_{2}}{\Longrightarrow} N_{\mathrm{amb}}=N_{\text {meas }} \cdot \frac{p_{1}}{p_{2}} \cdot \frac{T_{2}}{T_{1}}$.

As will be shown later on (cf. Sect. 2.4), the assumption that the particle mixing ratios within the flow field upstream of an underwing cloud probe remain constant (i.e. $\frac{n_{\mathrm{amb}}}{M}=\frac{n_{\mathrm{meas}}}{M}$ ) only partially reflects the reality. Essentially the particles' individual inertia causes a modification of the measurable particle mixing ratio compared to ambient state, which is generally of increasing importance with increasing particle size (mass) and with the increase of the aircraft's flight speed.

\subsection{TAS-based particle number concentrations}

If the air speed at the probe $\left(v_{2}\right)$ during measurements is unknown, e.g. for the case that the probe is not equipped with a pitot tube or when a present pitot tube is frozen, it is common practice to make the generalised presumption that the particle speed $\left(v_{\mathrm{p}}\right)$ equals the TAS $\left(v_{1}\right)$ to determine particle number concentrations (cf. Eq. 1).

Equivalent to using the TAS, the same resulting concentration is achieved when alternatively using the velocity ratio $\frac{\text { PAS }}{\text { TAS }}$ (i.e. $\frac{v_{2}}{v_{1}}$ ) as the factor for multiplication with measured particle number concentration $\left(N_{\text {meas }}\right.$, cf. Eq. 1), i.e.:

$N_{\text {meas }} \cdot \frac{v_{2}}{v_{1}}=\frac{n}{A_{\mathrm{s}} \cdot t \cdot v_{2}} \cdot \frac{v_{2}}{v_{1}}=\frac{n}{A_{\mathrm{s}} \cdot t \cdot v_{1}}$.

If pitot tube measurements of PAS are available, the treat-

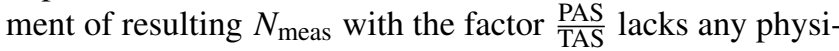
cal rationale and relies only on the geometrical consideration that $V_{1} \cdot t^{-1}=\mathrm{TAS} \cdot A_{\mathrm{s}}$ and that $V_{2} \cdot t^{-1}=\mathrm{PAS} \cdot A_{\mathrm{s}}$. Nevertheless, as both procedures yield identical results with the same error level, in the following the use of TAS for determining a particle number concentration is treated synonymously to correcting $N_{\text {meas }}$ (as defined in Eq. 1) by the factor PAS .

This approach results in significantly underestimated particle number concentrations with respect to the ambient conditions for following reasons:

1. By presuming $v_{1}$ as the speed of air while penetrating $A_{\mathrm{S}}$ it is insinuated that a certain number of particles per $\Delta t$ was detected while probing a linearly enlarged air volume per $\Delta t$. The compression of air causes a deceleration of the air flow upstream of an underwing probe. 
Thus, if based on $v_{1}$, the air volume probed per time interval is overestimated as the volumetric compression occurring in reality is not accounted for.

2. The dynamic pressure proportion gauged by means of pitot tube sensors is the measure of the air speed towards the pitot tube. Thus, the ratio of air speeds $\frac{\text { PAS }}{\text { TAS }}$ solely results from the dynamic pressure proportions, or rather the ratio thereof, obtained from two different sensors, the data boom and the instrument's pitot probe. The compression upstream of the instrument, however, predominantly impacts the absolute pressure at the point of measurement in comparison to ambient conditions. This means that the difference between the ambient state and measurement conditions, i.e. the compressed state of air within the detection region, is not accounted for by the ratio $\frac{P A S}{T A S}$.

Indeed, it needs to be taken into account that for susceptible particles the compression of air upstream of the probe induces changes in a particles' motion out of the initially undisturbed ambient state, e.g. if the particles are small enough such that they exhibit sufficient mobility. Thus, at the point of detection the changed particle motion excludes the nonrestrictive use of $v_{1}$ to describe the particle velocity through the detection region of the instrument.

Instead, dependent on their size (mass), the particles can be assumed to pass the instruments' detector with a velocity $\left(v_{\mathrm{p}}\right)$ that ranges between $v_{1}$ and $v_{2}$ (i.e. $v_{1} \leq v_{\mathrm{p}} \leq v_{2}$ ), while $v_{2}$ is generally smaller (by up to $30 \%$ ) than $v_{1}$. Strong indications for the trustworthiness of recorded $v_{2}$ (PAS) are given by the imaging technique of CIP-type instruments (also referred to as OAPs - optical array probes). This instrument type records image slices by means of a linear diode detector for subsequent reassembling to full 2-D images of respective particles. The scanning frequency and imaging rate of the linear diode detector is triggered by the air speed measured by the probe's pitot tube. Consequently, a significant deviation or falsification of this PAS measurement would result in distorted images. Laboratory calibrations are regularly performed by using a spinning disc of known rotation speed. Non-transparent circular spots on the disk are moved through the instruments sample area to simulate penetrating particles. The calibrations at relatively low penetration speeds $(\sim 23-$ $25 \mathrm{~m} \mathrm{~s}^{-1}$ ) compared to airborne measurements reveal that a deviation of the probe-measured air speed considerably exceeding $10 \%$ relative to the disc speed already causes a visible deformation of taken images as illustrated in Fig. 2. The strength of image distortion as a function of air speed deviation can be expressed by the aspect ratio of the taken images (Fig. 3) from a circular object that penetrates the instrument's detection region when the probe is calibrated with the spinning disc. The relationship between the image aspect ratio and the percentage of PAS deviation is almost linear, which appears to be plausible as the diode array scanning frequency should be proportional to the values of PAS. Thus the devi-

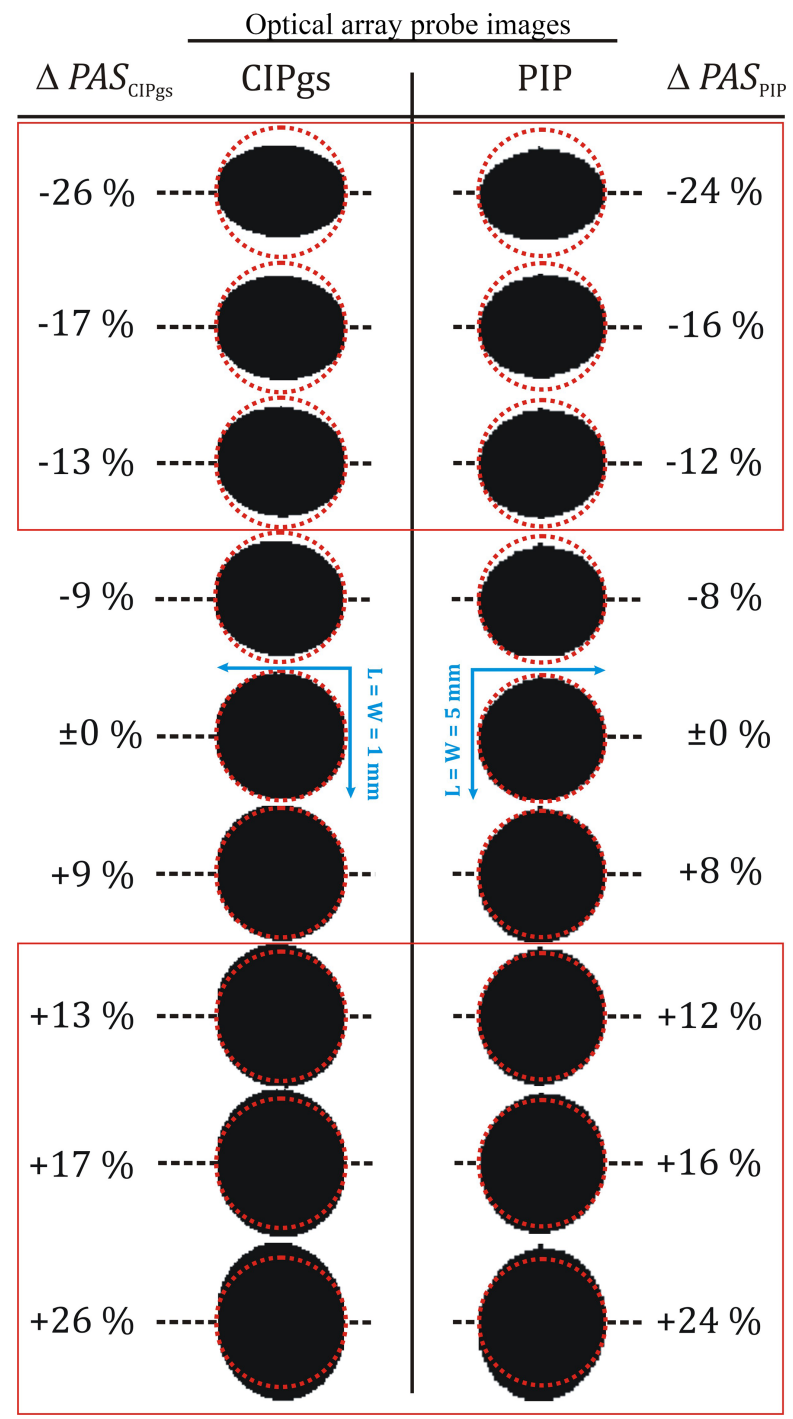

Figure 2. Shadow cast images of non-transparent circular spots on a spinning disk (for calibration purposes) passing the probes' sample area $A_{\mathrm{s}}$ with constant velocity $\left(\sim 23-25 \mathrm{~m} \mathrm{~s}^{-1}\right)$. For this illustration, in the data acquisition program, the probe air speed (PAS) is manually varied stepwise with the finest available resolution for triggering the timing of imaging. Manually shifting the PAS causes a positive or a negative deviation $(\triangle \mathrm{PAS})$ of the air speed relative to the constant disk rotation speed $v_{\text {Rot }}$. Deformed images relative to the dashed red circles of identical diameter (according to $\triangle \mathrm{PAS}= \pm 0 \%$ if $v_{\text {Rot }}=\mathrm{PAS}$, marked in blue) indicate image distortion that becomes significant for $\triangle \mathrm{PAS}$ exceeding $10 \%$ (red boxes). PIP images are slightly shifted as $v_{\text {Rot }}$ of two radially opposed points on the edges of a $5 \mathrm{~mm}$ sized disk spot increases with distance from the disk's centre.

ation between PAS and particle penetration speed exhibits a linear relationship with the image aspect ratio. Appropriate analyses of images taken from initially spherical particles, i.e. from droplets, may suffice for qualitatively evaluating measured PAS compared to the factual particle penetration 


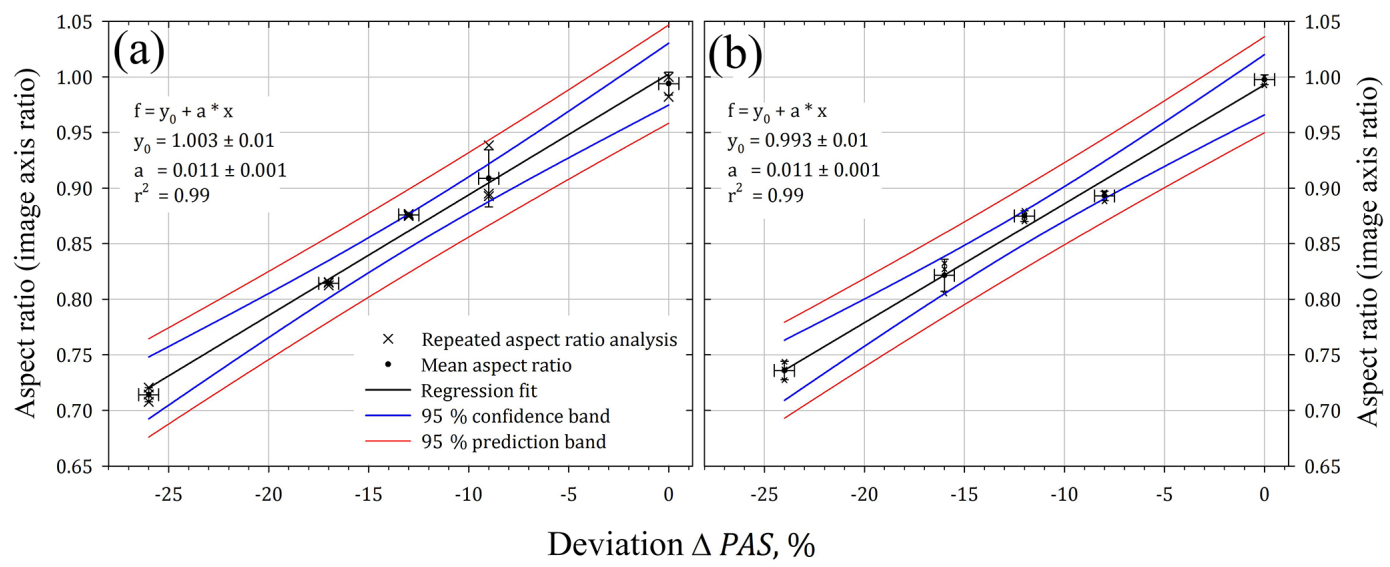

Figure 3. Aspect ratios of taken images as a function of the deviation of the probe air speed (PAS) from the penetration speed of a circular object through the instrument's detection region. The image aspect ratio provides a measure of the distortion strength when the PAS setting is manually shifted in the data acquisition software compared to the constant penetration speed of a circular object on the spinning disc used for calibrations of an optical array probe (OAP). (a) The Cloud Combination Probe's CIPgs; (b) the Precipitation Imaging Probe (PIP).

speed. At higher air speeds (e.g. up to $250 \mathrm{~m} \mathrm{~s}^{-1}$ for $H A L O$ ) it is expected that even smaller uncertainties of measured PAS related to the true particle penetration speed cause severe distortion of resulting images. Thus, for measured $v_{2}$ we assume the uncertainty to range within $\sim 10 \%$ if recorded particle images of droplets do not systematically exhibit a strong and, therefore, obvious deformation.

\subsection{The cloud particles' mobility}

The ability of particles to adapt to changes in air speed, the particles' mobility, depends on the particle size, mass and thus inertia and can be expressed by the calculable relaxation time (Hinds, 1999; Kulkarni et al., 2011, and Willeke and Baron, 1993, respectively). Cloud particles, for example, of sizes smaller than $100 \mu \mathrm{m}$ diameter, moving with $70 \mathrm{~m} \mathrm{~s}^{-1}$ at atmospheric pressures of $300 \mathrm{hPa}$ and temperatures of $240 \mathrm{~K}$, have relaxation times (at the most 9 milliseconds) on the same order of magnitude as the compression timescales (24 milliseconds at flight speeds from 125 to $250 \mathrm{~m} \mathrm{~s}^{-1}$; cf. Sect. 3.2). To assess the ability of larger cloud particles to adapt to changes in air speed, about 400 particle images from Cloud Imaging Probe greyscale (CIPgs) measurements over two flights "AC07" and "AC13" during the HALO mission ACRIDICON-CHUVA (for further details see Sect. 3.4) were analysed. The aspect ratio of images taken from presumably spherical cloud particles was charted as a function of particle size. The images of spheroidal objects were selected by means of an automated image analysis algorithm after carefully filtering the particle images with respect to the atmospheric conditions during measurement and appearance (for details cf. Appendix B). Generally, only images were analysed with image geometries greater than $75 \mu \mathrm{m}$ in diameter (along both directions of the image's main axes). Most of the analysed particle images exhibit a Poisson spot as a result of Fresnel diffraction (Korolev, 2007). With increasing distance $\left(Z_{\mathrm{d}}\right)$ of a particle from the object plane when penetrating the OAP detection region:

1. the size of resulting Poisson spot increases and

2. the optical aberration is amplified; i.e. the particle image exhibits an expansion compared to the true dimension of the detected cloud element.

The cloud droplet size is reconstructed from the image dimension in relation to the Poisson spot's size as described by Korolev (2007) - thus, reproduced particle sizes may be smaller than the $75 \mu \mathrm{m}$ threshold for the image size. Particle images emanating from cloud elements which passed the detection region in a distance that was too far away from the object plane $\left(Z_{\mathrm{d}}>4\right)$ were discarded from further analysis. The automated analysis is based on the Bresenham algorithm (Bresenham, 1965), approximating an ellipse to the shape of the particle images (of $15 \mu \mathrm{m}$ image resolution) that remain after the selection process. The 395 selected particle images of the data set of two flights that fulfil the criteria to be further analysed are then charted in terms of image aspect ratio as a function of reconstructed particle size. In fact, the lengths of the main axes of the approximated ellipse are used to determine the aspect ratio of the individual image.

Figure 4a depicts the result of this analysis. The scatter of the single data points (Fig. 4a) is statistically processed by calculating the aspect ratio median with percentiles $(10,25$, 75 and $90 \%$ ) in particle diameter size bins of $15 \mu \mathrm{m}$ (Fig. 4b). Images of particles with diameter smaller than $70 \mu \mathrm{m}$ show distortions of about $13 \%$, which is synonymous with a $v_{\mathrm{p}}$ PAS - deviation of less than $13 \%$ (cf. Fig. 3). For droplets of diameter between 70 and $100 \mu \mathrm{m}$ the image aspect ratios increasingly scatter, but the resulting median does not indicate that $v_{2}$ deviates from PAS by more than $20 \%$. The im- 


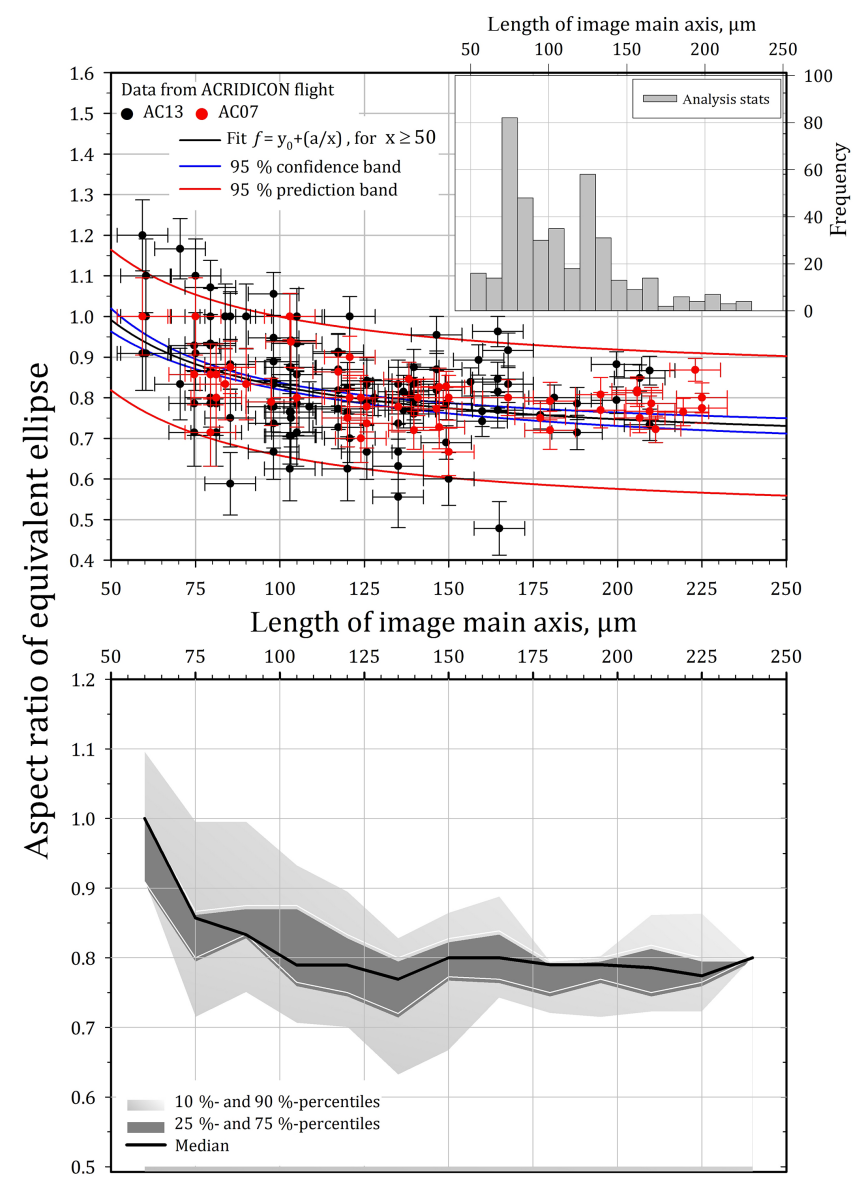

Figure 4. Aspect ratios as a function of the image's main axis dimension as revealed from the automated reanalysis of $\sim 395$ individual particle images acquired by the CCP during two ACRIDICON-CHUVA mission flights, "AC07" on 6 September and "AC13" on 19 September 2014, over the Amazonian basin, Brazil. The automated procedure to identify spherical particles and to determine their images' aspect ratio by means of the equivalent-ellipse approximation is described in detail in Appendix B. (a) The determined aspect ratio of the individual images of spherical particles and (b) the statistically analysis provided as median of the aspect ratios together with $10 \%, 25 \%, 75 \%$ and $90 \%$ percentiles.

ages of particles with diameter larger than $100 \mu \mathrm{m}$ exhibit increasing distortion as the image aspect ratios approach values, suggesting a $v_{\mathrm{p}}$-PAS deviation of up to $20 \%$. The observations indicate that the driving forces arising in the flow field upstream of an underwing probe overcome the inertia resistance of small cloud elements $\left(D_{\mathrm{p}}<70 \mu \mathrm{m}\right)$ but not of larger cloud elements of diameter larger than $100 \mu \mathrm{m}$. This supports the suggestion that the penetration speed of the vast majority of detected particles through an OAP's detection region may be best described by PAS $<v_{\mathrm{p}}<\mathrm{TAS}$. Thus, beside the correction to account for the compression, a further correction concerning the particle inertia may be applied to resulting particle number concentrations.
CFD simulations of the pressure field and resulting droplet trajectories in the close vicinity of an OAP instrument (CIP, cf. Sect. 3.1) were performed (using CFX 17.0 by ANSYS Inc.). For this investigation the aircraft's structure as well as the particular geometry of the $H A L O$ underwing probe configuration had to remain unconsidered (cf. Sect. 1). With respect to the flow field boundaries a comparatively large model domain was initialised with edge lengths of 15 times the instrument's width and 15 times the instrument's height. The model's mesh comprised $2.17 \times 10^{6}$ nodes and $8.2 \times 10^{6}$ elements containing 15 inflation layers. The flow field was calculated by solving the Navier-Stokes equation for a steady state, compressible (transport of enthalpy including the kinetic energy effects) and turbulent flow. The shear stress transport $k-\omega$-based turbulence model (e.g. Menter et al., 2003) was used. The calculations were set up with a turbulence intensity of $5 \%$. The particle trajectory calculations, relying on the Schiller-Naumann drag force model (cf. Naumann and Schiller, 1935), were decoupled from the continuous fluid simulation as for the simulations any influence of the very few particles on the flow field was assumed to be negligible. Particles' bouncing from walls was excluded as well as any turbulent dispersion force. The simulations were initialised with typical conditions as encountered throughout a $(H A L O)$ measurement flight. One set of atmospheric conditions under undisturbed ambient conditions was used as the simulation input, for example $409 \mathrm{hPa}\left(p_{1}\right)$ and $241 \mathrm{~K}$ $\left(T_{1}\right)$. Additionally, a typical TAS of $187 \mathrm{~m} \mathrm{~s}^{-1}$ (Mach 0.6) was taken from the recorded data set to initialise the particle trajectory analyses. In the initial state the cloud droplets of various diameters $(5,50,70$ and $100 \mu \mathrm{m})$ were assumed to move towards the instrument with a speed equal to TAS. The simulation of the pressure field (Fig. 5) qualitatively supports the observation that the air is compressed in the OAP's detection region (blue circle) in comparison to the ambient state. Moreover, the simulations qualitatively agree with the assumption that the particles' speed is changed compared to their initial speed when they pass the plane of the OAP's detection region. However, as will be shown later (cf. Sect. 3.2), quantitatively the simulation exhibits significant deviations from the in-flight measured compression strength and thus the strengths of particle deceleration. The simulation, for example, forecasts a pressure increase in the detection region by about $5.5 \%$ compared to ambient conditions. Contrarily, the comprehensive data sets of three independent underwing cloud probes at similar flight speeds consistently indicate a pressure increase of about $10 \%$ (cf. Figs. 8 and 9). Furthermore, the simulated cloud droplet deceleration is not as large $\left(12,3,2\right.$ and $1 \%$ for $D_{\mathrm{p}}$ of $5 \mu \mathrm{m}, 50,70$ and $100 \mu \mathrm{m}$, respectively) as suggested by the grade of image distortion. However, it needs to be noted that these simulations comprise one particular case of atmospheric conditions with the idealising assumption that the instrument is aligned isoaxially with the air flow. Small changes of the aircraft's angle of attack may significantly modify the pressure field upstream of 
the cloud probe compared to the CFD simulation of this particular idealised case. Moreover, any further obstacle to the air flow (e.g. the aircraft's wing, the neighboured underwing cloud probe or the pylon) is not taken into consideration by this CFD simulation. Thus it is conceivable that these simulations do not sufficiently reflect the real measurement conditions. More comprehensive CFD analyses should doubtlessly also consider variable atmospheric conditions, the aircraft's geometry and the variable aircraft's attitude during flight as well as an adjacent flow obstacle. These new CFD simulations should also account for different particle sizes of both phases (liquid and frozen), all of which may be coverable only by a separate study as it exceeds the scope of this publication.

Hence, we conclude that

1. there are strong indications that the compression of air causes a densification of small airborne particles $\left(D_{\mathrm{p}}<70 \mu \mathrm{m}\right)$ in the detection region of the considered instrument;

2. for these small particles $\left(D_{\mathrm{p}}<70 \mu \mathrm{m}\right)$ the compression is reflected by systematically lower values of $v_{2}$ (PAS) compared to $v_{1}$ (TAS), exhibiting a discrepancy that is too large to be covered by a $10 \%$ uncertainty of measured PAS;

3 . for these small particles $\left(D_{\mathrm{p}}<70 \mu \mathrm{m}\right)$ the particles' velocity $v_{\mathrm{p}}$ while passing the instrument sample area is best approximated with $v_{2}$, rather than with $v_{1}$, whilst for larger particles $\left(D_{\mathrm{p}}>100 \mu \mathrm{m}\right)$ the inertia forces lead to a particle velocity of PAS $<v_{\mathrm{p}}<\mathrm{TAS}$;

4. the conventionally applied practice of treating $N_{\text {meas }}$ with the factor $\frac{\text { PAS }}{\text { TAS }}$ (geometric approach) is invalid for correcting measured number concentration to ambient conditions as the air volume probed per time interval differs under compressed conditions from the air volume under ambient conditions;

5. a method is needed to reasonably correct $N_{\text {meas }}$ by accounting for the air compression, particularly at high flight velocities, to determine $N_{\mathrm{amb}}$;

6. an additional correction is needed that accounts for the inhibited mobility of larger cloud droplets $\left(D_{\mathrm{p}}>100 \mu \mathrm{m}\right)$ resulting in a penetration speed through the OAP detector, which ranges from PAS $<v_{\mathrm{p}}<\mathrm{TAS}$, whereas the assumption $v_{\mathrm{p}} \approx \mathrm{PAS}$ is invalid for droplets of this size.

\subsection{Correction of $N_{\text {meas }}$ based on thermodynamic considerations}

The expression that accounts for the described compression effect is formulated in its general form with Eq. (5). The unknown parameter in this expression is the probe air tempera-
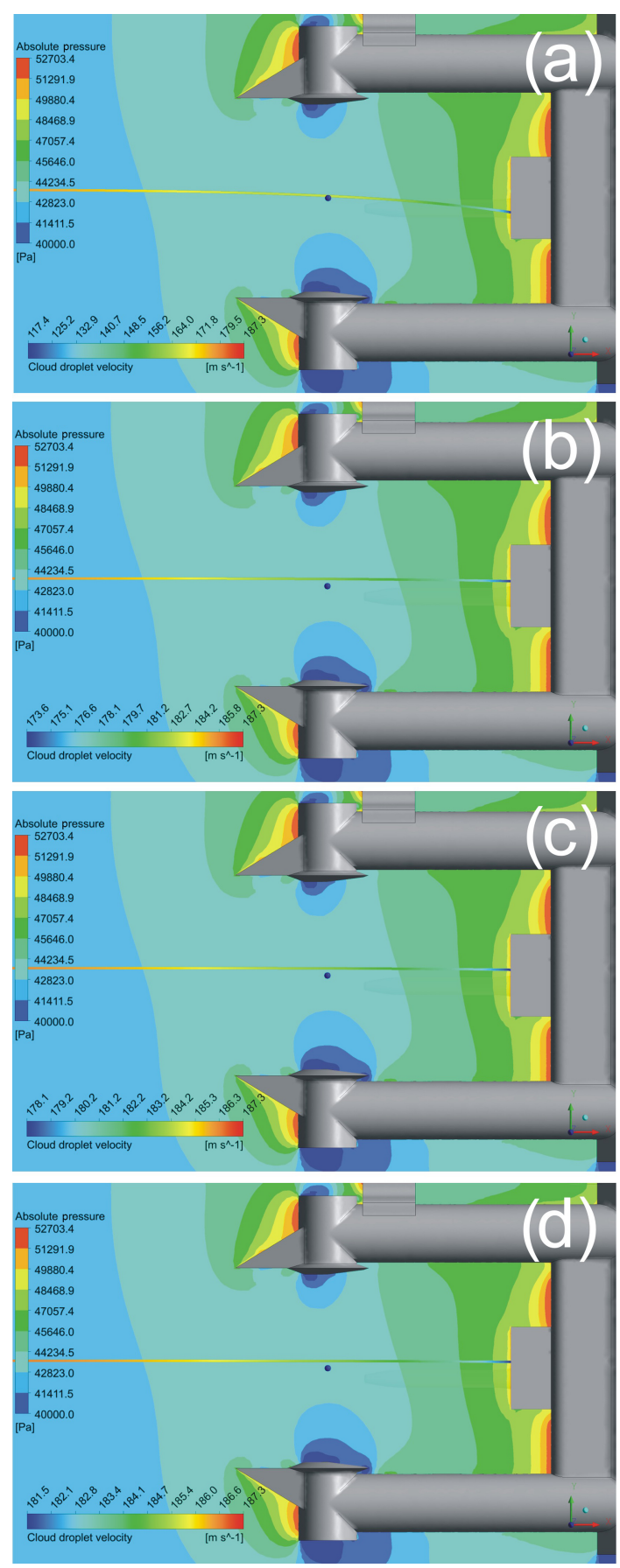

Figure 5. Computational fluid dynamic (CFD) simulations of the pressure field upstream of a CIP-type instrument head for one initial state at $p_{1}=409 \mathrm{hPa}, T_{1}=241 \mathrm{~K}$ and TAS $=v_{\mathrm{p}}=187.29 \mathrm{~m} \mathrm{~s}^{-1}$. Background colour contours indicate the change of absolute pressure upstream of the probe. The plane of detection region is signified by a single bluish circle. Cloud particle trajectories were simulated for different droplet sizes: (a) $D_{\mathrm{p}}=5 \mu \mathrm{m}$, (b) $D_{\mathrm{p}}=50 \mu \mathrm{m}$, (c) $D_{\mathrm{p}}=70 \mu \mathrm{m}$ and (d) $D_{\mathrm{p}}=100 \mu \mathrm{m}$, illustrated by their trace through the detection region. The change of the particles' speed towards the instrument is colour-coded along the trace correspondingly to the generally decreasing cloud droplet velocity. 
ture $T_{2}$ that is increased in comparison to ambient air temperature $T_{1}$ as a consequence of the compression. The temperature increase is obtainable by using Bernoulli's law together with the ideal gas law and, furthermore, by presuming adiabatic conditions, i.e. the conservation of energy. The derivation emanates from following different conditions which are illustrated in Fig. 1 for the air velocity $v$, the air pressure $p$, the specific enthalpy $h$ of an uniform system and the gravitational potential $\phi$ : based on Bernoulli's law for compressible gases the specific enthalpy is transduced into an expression that includes the air's specific heat capacity $c_{\mathrm{p}}$ (presumed as nearly constant under most of the flight conditions; cf. Appendix A) and the temperatures states in the ambient environment and under measurement conditions of the OAP detection region. Solving the equation for the probe air temperature $T_{2}$ finally allows for converting Eq. (5) into the aspired expression:

$$
\begin{aligned}
N_{\mathrm{amb}} & =N_{\text {meas }} \cdot \frac{p_{1}}{p_{2}} \cdot\left(1+\frac{1}{2008 \mathrm{~J} \mathrm{~kg}^{-1} \mathrm{~K}^{-1} \cdot T_{1}}\left(v_{1}^{2}-v_{2}^{2}\right)\right) \\
& =N_{\text {meas }} \cdot \xi .
\end{aligned}
$$

For the detailed steps taken to derive Eq. (7) refer to Appendix A.

\subsection{Correction for the particle inertia}

Apart from the compression correction one further correction needs to be considered that results from the inertia of probed cloud elements. As illustrated in Sect. 2.2, small cloud particles $\left(D_{\mathrm{p}}<70 \mu \mathrm{m}\right)$ exhibit enough mobility to adapt a movement into flight direction that causes the particles to penetrate the OAP detection region with $v_{\mathrm{p}} \approx \mathrm{PAS}$. The same investigation shows that all detected presumably liquid spheroids with diameter $100 \mu \mathrm{m}<D_{\mathrm{p}}<225 \mu \mathrm{m}$ penetrate the detection region with $v_{\mathrm{p}}$ that is about $20 \%$ faster than the PAS but still about $10 \%$ slower than the measured TAS. Hence, the assumption of $v_{\mathrm{p}} \approx$ PAS seems to lack further validity for particles with $D_{\mathrm{p}}>100 \mu \mathrm{m}$, whereas more likely a penetration speed of PAS $<v_{\mathrm{p}}<$ TAS is to be presumed for particles of this size. As an example, this means that compact cloud elements of size $100 \mu \mathrm{m}<D_{\mathrm{p}}<225 \mu \mathrm{m}$ for which systematically distorted images are recorded (with aspect ratio of $\sim 0.8$; $\mathrm{cf}$. Fig. 4) penetrate the OAP detection region faster (by $\sim 17-$ $20 \%$, cf. Fig. 3) than the surrounding air at the point of measurement. In other words, due to the particles' individual inertia, the large and compact cloud elements $\left(D_{\mathrm{p}}>100 \mu \mathrm{m}\right)$ are over-represented in the detection region compared to ambient conditions. Thus, for larger cloud droplets the particles' size (inertia) needs to be considered with the additional correction $\mu$ as a function of $D_{\mathrm{p}}$ such that Eq. (7) in generalised form reads as

$N_{\mathrm{amb}}=N_{\text {meas }} \cdot \xi \cdot \mu\left(D_{\mathrm{p}}\right)$.

Accepting that for small cloud elements $\left(D_{\mathrm{p}}<70 \mu \mathrm{m}\right)$ the inertia is negligible due to the particles' mobility, a general cor- rection with $\mu=1$ in most cases may suffice such that $N_{\mathrm{amb}}$ is determined as suggested by Eq. (7). The previous investigation concerning the grade of distortion of recorded particle images (Sect. 2.2) suggests that in this particular case for larger cloud droplets $\left(100 \mu \mathrm{m}<D_{\mathrm{p}}<225 \mu \mathrm{m}\right)$ an inertia correction with $\mu=0.8$ is appropriate.

Thus, in summary, an inertia correction with $\mu$ may be applied by following particle-size-dependent first-order approach:

$\mu\left(D_{\mathrm{p}}\right)=\left\{\begin{array}{l}1 \text { for cloud particles of diameter } D_{\mathrm{p}}<70 \mu \mathrm{m} \\ 0.8 \text { for compact particles, with } 100 \mu \mathrm{m}<D_{\mathrm{p}}<225 \mu \mathrm{m} .\end{array}\right.$

As corresponding data sets are lacking for larger-sized cloud and precipitation droplets $\left(225 \mu \mathrm{m}<D_{\mathrm{p}}<6 \mathrm{~mm}\right)$ the results of available data sets could be extrapolated towards larger particle sizes. With increasing size of liquid droplets, i.e. in the millimetre size range, any unbiased image aspect ratio analysis is impeded as these precipitating droplets are increasingly impacted to deviate from spherical shapes (Thurai et al., 2009). However, it remains only to surmise that an inertia correction between 0.8 and 0.7 is to be applied to liquid cloud elements and precipitating droplets of diameter $225 \mu \mathrm{m}<D_{\mathrm{p}}<6 \mathrm{~mm}$.

For spherical ice particles further differentiation is required concerning the definition of particle diameter. In the liquid phase the geometrical diameter (equivalent to the herein used $\left.D_{\mathrm{p}}\right)$ and the aerodynamic diameter $\left(D_{\mathrm{a}}\right)$ of a particle are almost identical $\left(D_{\mathrm{p}} \approx D_{\mathrm{a}}\right.$ with $D_{\mathrm{a}}=0.99 \cdot D_{\mathrm{p}}$, for atmospheric air pressures of 1000-200 hPa) (Kulkarni et al., 2011; Willeke and Baron, 1993). The $D_{\text {a }}$ of a frozen spheroid, however, significantly differs from corresponding geometrical diameter $\left(D_{\mathrm{a}}=0.95 \cdot D_{\mathrm{p}}\right.$, with negligible uncertainty over the range of $1000-200 \mathrm{hPa}$ ). This means that frozen spheres of any geometrical size $D_{\mathrm{p}}$ exhibit the aerodynamic behaviour, i.e. the mobility, of a less inert particle of smaller size, which is expressed by $D_{\mathrm{a}}$. Thus, for particles of geometrical sizes $70 \mu \mathrm{m}<D_{\mathrm{p}}<100 \mu \mathrm{m}$ an inertia correction of general validity is difficult to specify. For this particular size range, the value of $\mu$ may be closer to 1 in cases when the particles are frozen as their aerodynamic diameters correspond to smaller equivalent sizes (e.g. $66.5 \mu \mathrm{m}<D_{\mathrm{a}}<95 \mu \mathrm{m}$, at $200 \mathrm{hPa}$; Kulkarni et al., 2011; Willeke and Baron, 1993). Frozen cloud elements exhibit reduced material density (mass) and thus reduced compactness (if ice particles exhibit extremities) compared to a compact (liquid) cloud droplet of the same geometrical diameter. As a consequence, grown ice particles (e.g. needles, plates, dendrites, bullets or higher-grade combinations) may exhibit lower inertia, yielding $\mu$ values above 0.8 . Moreover, the mobility of larger ice particles, i.e. with $D_{\mathrm{p}}>100 \mu \mathrm{m}$, may improve due to a diminishing inertia effect when these particles form extremities resulting in $\mu$ values well between 0.8 and 1.

Furthermore, in the progressed state of ice formation certain disturbances of the local flow field in the vicinity of 
underwing probes can cause an almost uniform population of ice particles (either predominantly columnar or planar) to pass the OAP detection region with a preferential orientation (cf. Wendisch and Brenguier, 2013; Sect. 6.4.1.2 therein). Such a preferential orientation would be visible in recorded 2-D images of these particles, exhibiting shapes that are systematically aligned. This effect is indicative of an impact of the local flow field on the ice particles' airborne state while passing through the detection region.

In essence, any inertia correction turns out to be individually applicable after careful investigations with respect to the particles' phase and nature (i.e. density, compactness, shape) during the flight. For data obtained exclusively from iced clouds, e.g. from measurements in cirrus clouds or in the outflow anvil of a convective cloud system, it appears suitable to generally assume an inertia correction with $\mu$ values above 0.8. Corrections of measured cloud particle number concentrations accounting for the particles' inertia need to be applied carefully. In contrast, the air's compressibility is generally a feature to be considered which is of increasing importance with the aircraft's flight velocity. Compared to the undisturbed ambient state, the compression of the probed air volume upstream of a flow obstacle exhibits a varying but continuous impact on the particle number concentrations to be measured. Therefore, for any underwing probe measurement aiming at cloud element number concentrations under ambient conditions, the compression of the probed air volume compared to the ambient state needs a non-restrictive correction as a function of flight velocity.

\section{Applying the compression correction to airborne measurements}

The magnitude of compression increases with air speed, i.e. with flight velocity. Thus, the derived thermodynamic correction should have the largest effect on data acquired during flights with fast aircraft, for example with the Learjet-35A, the Gulfstream G-550 HALO or HIAPER (up to Mach 0.75, corresponding to $\sim 250 \mathrm{~m} \mathrm{~s}^{-1}$ ). The extent of such corrections underlying both the geometric and the thermodynamic perspective, and their impact on the measured data, is discussed in the following for actual measurements from three (out of eight) underwing probes deployed on the HALO aircraft.

\subsection{Instrumentation related to cloud particle microphysics}

The three selected instruments are PMS-type underwing probes which are commercially available from the instrument manufacturer Droplet Measurement Technologies (DMT, Boulder, CO, USA) with the general purpose of investigating the microphysical properties of cloud elements and hydrometeors. One particular measurement technique the three instruments have in common is based on the principle of OAPs as described by Knollenberg (1970). Advanced developments of the OAP measurement method led to the shadow cast imaging instruments of different types (Korolev et al., 1991, 1998; Korolev, 2007; Lawson et al., 2006) that are currently in use. The $H A L O$ underwing probes to be discussed are as follows.

1. The CCP combines two detectors:

a. the Cloud Droplet Probe (CDP), detecting forward scattered laser light due to particles penetrating the CDP detection area (Lance et al., 2010) as an advanced development of the Forward Scattering Spectrometer Probe (FSSP) technique (cf. Dye and Baumgardner, 1984; Baumgardner et al., 1985; Korolev et al., 1985);

b. the CIPgs, which records 2-D shadow cast images of cloud elements that cross the individual CIPgs detection region.

CCP measurements overall cover a particle diameter size range from 2 to $960 \mu \mathrm{m}$. The performance of the specific CCP instrument used in this study is demonstrated by earlier investigations related to clouds in the tropical convective outflow (Frey et al., 2011), concerning polar stratospheric clouds (Molleker et al., 2014) or within low-level mixed-phase clouds in the Arctic (Klingebiel et al., 2015) when deployed at much slower flight velocities $\left(<170 \mathrm{~m} \mathrm{~s}^{-1}\right)$.

2. The Novel Ice eXpEriment - Cloud, Aerosol and Precipitation Spectrometer (NIXE-CAPS) described by Meyer (2012) also combines two measurement techniques:

a. The CAS-DPOL module (Cloud and Aerosol Spectrometer) is based on the principle of forward scattering detection similar to the CDP (cf. above) but, instead of using an open-path detection region (CDP), the CAPS is equipped with an inlet tube. In addition, the CAS-DPOL discriminates between spherical and aspherical particles by measuring the change of polarisation of laser light that is scattered by single particles (cf. Baumgardner et al., 2001, 2014).

b. Additionally, NIXE-CAPS is equipped with a CIPgs instrument (cf. CCP).

With NIXE-CAPS cloud particles with diameters between 0.6 and $\sim 950 \mu \mathrm{m}$ are detected. Note that the thermodynamic correction derived here applies as such to particle number concentrations measured particularly with the OAPs (the CIPgs probes and the PIP) since the flow conditions inside the inlet tube of the CAS-DPOL differ from those of the open-path instruments. 
Cloud Combination Probe (CCP)

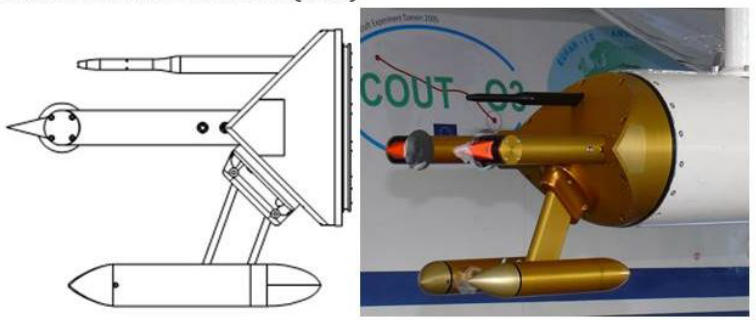

Novel Ice eXpEriment - Cloud, Aerosol and Precipitation Spectrometer (NIXE-CAPS)
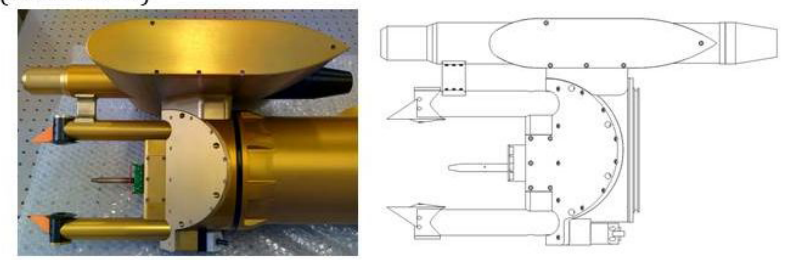

Precipitation Imaging Prope (PIP)
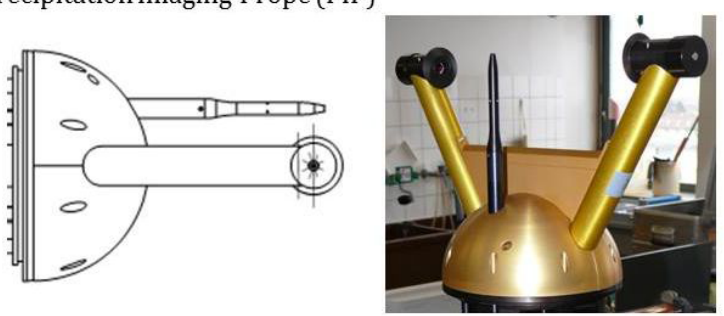

Figure 6. Diagrams and images of the different instrument heads of quasi-streamlined design. Top: Cloud Combination Probe (CCP) with $90^{\circ}$ angled wedge. Middle: combined probe head of $90^{\circ}$ angled wedge and additional winglet of NIXE-CAPS. Bottom: Precipitation Imaging Probe (PIP) with half-sphere probe head.

3. The PIP detects precipitating cloud elements and hydrometeors by means of particle-induced shadow projection onto a diode sensor, allowing for a 2-D particle imaging similar to the CIPgs. In comparison to the CIPgs, the PIP setup features an increased detection volume that covers larger particle sizes with $100 \mu \mathrm{m}<D_{\mathrm{p}}<6400 \mu \mathrm{m}$.

One major difference between CCP, NIXE-CAPS and PIP is the instrument-specific design of the probe heads. As shown in Fig. 6, CCP is equipped with a $90^{\circ}$ angled wedge. NIXE-CAPS combines a wedge of the same shape with an additional aerodynamic winglet that may significantly contribute to the effective cross-sectional area of the NIXECAPS body. PIP is equipped with a half-sphere front cap. The different instrument heads have individual extension tips. Between the tips a free laser beam crosses the freely flowing sample air through which the particles pass. The sample area $A_{\mathrm{s}}$ of the probes, where the instrument is sensitive for particles crossing the open laser beam, is located almost half way between the tips.
One further important difference of the three instruments is their mounting position on $H A L O$ with respect to the aircraft fuselage (cf. Fig. 1b). PIP is mounted closest to the aircraft fuselage under the portside wing. NIXE-CAPS and CCP are positioned under the starboard wing on the intermediate and outbound hardpoints, respectively. Thus, with the three selected instruments the full range of available underwing probe positions with respect to the aircraft fuselage of $H A L O$ is covered.

\subsection{Specific correction factor $\xi$ for $H A L O$ instruments}

The continuous measurements of the parameters $v_{1}$ (TAS), $v_{2}$ (PAS), $T_{1}$ (static air temperature), $p_{1}$ (static ambient pressure) and $p_{2}$ (static pressure at the probes) during flight allow for deriving the factors for the geometric correction $\frac{\text { PAS }}{\text { TAS }}$ and the thermodynamic correction $\xi$ as a function of TAS with $1 \mathrm{~Hz}$ temporal resolution. Hence, individual $\xi$ corrections are obtainable at any time during the measurement with pitot-equipped instruments. Figure 7 shows the comparison of calculated $\xi$ and $\frac{\text { PAS }}{\text { TAS }}$ corrections (synonymous for using TAS instead of PAS for $N_{\text {meas }}$, cf. Sect. 2.1) as a function of TAS. The unadjusted data from 6 out of a total of $11 \mathrm{ML}$ CIRRUS flights are shown. In sum, for the following, the $1 \mathrm{~Hz}$ resolved data of more than 35 flight hours are treated.

It needs to be noted that throughout the ML-CIRRUS measurements the PAS of the PIP was systematically affected due to the malfunction of a temperature sensor; i.e. a broken temperature sensor in the PIP's pitot tube provided a constantly false temperature output of about $320 \mathrm{~K}$. This caused a false measurement of the PIP's PAS which, however, never exceeded a $10 \%$ deviation compared to the PAS measured by the other cloud probes. Thus, the resulting falsification of the PIP's PAS was reconstructed by adopting exclusively the missing temperature data from a pitot tube of another underwing probe (e.g. the CCP) which was functioning normally. The reconstruction of the PIP's PAS - still comprising the PIP's own pressure measurements - may contain uncertainties. However, calculations show that temperature deviations of $\pm 20 \mathrm{~K}$ (as well as with $\pm 10 \mathrm{~K}$ ) employing the adopted temperature values cause the resulting PAS to vary by about $\pm 5 \%$ ( $\pm 3 \%)$. Thus, the uncertainty of the PIP's reconstructed PAS is minor compared to the uncertainty that is generally presumed for the PAS measurement which exhibits a significantly higher sensitivity to measured pressures.

During the flight on 29 March 2014 (red data points) the factors $\frac{\text { PAS }}{\text { TAS }}$ and $\xi$ as a function of TAS occasionally show significant deviation from the generally observed course. This deviation can unambiguously be apportioned to disturbed PAS measurements. The PAS chart is subject to disturbances either due to freezing conditions causing the pitot tube to be tamped or due to non-isoaxial airflow caused by flight manoeuvres like tight turns. Very few and relatively short periods of PAS disturbances also occurred during the flight on 11 April 2014 (pink data points). 


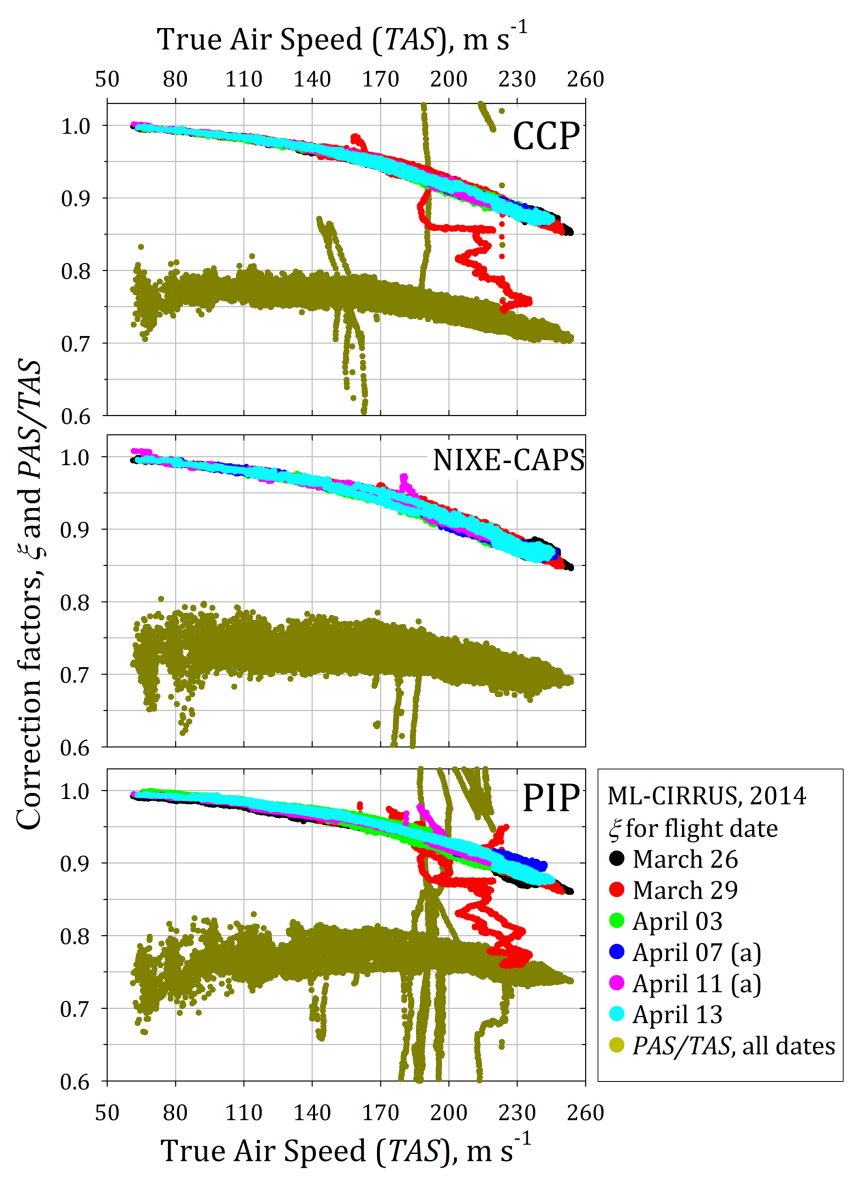

Figure 7. Comparison of two different instrument-specific corrections applied to data acquired on $H A L O$ during ML-CIRRUS. The geometric correction $\frac{\text { PAS }}{\text { TAS }}$ causes a general downscaling of measured concentrations of $20 \%$ up to $35 \%$ for NIXE-CAPS and CCP. Thereby $\frac{\text { PAS }}{T A S}$ is highly variable, ambiguous and shows certain dependences on the instrument's wing position. Instrument-specific $\xi$ values exhibit higher compactness over the TAS range and show reduced dependence on the wing position. The data are from six ML-CIRRUS flights and include outliers due to freezing of the pitot tubes or due to distortions from isoaxial flow accompanied with manoeuvres such as tight turns.

The $\xi$ correction is a monotonous function of flight velocity that has increasing effectiveness for each of the three instruments. Contrarily, the $\frac{\text { PAS }}{\text { TAS }}$ correction appears to be systematically effective over the full range of air speeds, even at the lowest aircraft velocities - while the scatter of $\frac{\text { PAS }}{\text { TAS }}$ by $\sim 2-10 \%$ may result from small-scale turbulence or nonisoaxial airflow. However, the geometric correction with $\frac{\text { PAS }}{T A S}$ causes a general reduction of the values measured with CCP and NIXE-CAPS of not less than $20 \%$, even reaching $35 \%$ for NIXE-CAPS (cf. Fig. 7). For CCP the values of $\xi$ and $\frac{\text { PAS }}{\text { TAS }}$ are most compact. The variability, in particular of $\frac{\text { PAS }}{\text { TAS }}$, increases for NIXE-CAPS over the complete TAS range. For PIP the $\xi$ factor is comparably variable at flight velocities greater than $140 \mathrm{~m} \mathrm{~s}^{-1}$.
The events when the pitot tube was frozen or affected by misaligned inflow (mainly attributed to the measurements made on 29 March 2014 and to a limited number of measurements made on 11 April 2014) were removed from the data set, which effectively reduces the data set volume by less than $3 \%$.

For CCP measurements (data set as treated for Fig. 7) the parameters for calculating $\xi$ according to Eq. (7) are shown in Fig. 8a as a function of TAS. Displayed are the absolute differences of measured pressures $\left(p_{1}, p_{2}\right)$ and velocities $\left(v_{1}\right.$, $v_{2}$ ). The difference of the squared velocities $v_{1}$ and $v_{2}$ (Eq. 7) is implicitly included in calculated $T_{2}$. Moreover, the difference between measured temperature $T_{1}$ and the calculated temperature $T_{2}$ is shown. At a maximum TAS of $255 \mathrm{~m} \mathrm{~s}^{-1}$ the compression impact causes a $\Delta v$ of up to $75 \mathrm{~m} \mathrm{~s}^{-1}$, a $\Delta T$ of up to $16 \mathrm{~K}$ and a $\Delta p$ of about $30-60 \mathrm{hPa}$. The values of $\Delta T$ and $\Delta p$ upstream of an underwing probe may appear surprisingly high but are largely consistent with the results of a fluid-dynamical simulation at similar flow velocities $\left(200 \mathrm{~m} \mathrm{~s}^{-1}\right)$ for another underwing probe geometry (Abdelmonem et al., 2016). Moreover, these observations are largely consistent with the results of theoretical considerations regarding the thermodynamic processes inherent with the compression of air (cf. Appendix A). In Fig. 8b the results of the pressure expression $\frac{p_{1}}{p_{2}}$ (green data points) and temperature fraction $\frac{T_{2}}{T_{1}}$ (black data points), as applied in Eq. (7), are displayed as a function of TAS, illustrating the respective effectiveness of each term to calculated $\xi$. The inversion to $N_{\text {amb }}$ causes $N_{\text {meas }}$ to be reduced by a factor of up to 0.8 to compensate for the induced pressure increase. In contrast, the compression-induced heating of air needs to be corrected by a factor of up to 1.07 .

Note that for a TAS $\mathrm{Tax}_{\max }$ of $255 \mathrm{~m} \mathrm{~s}^{-1}$ the compressioninduced heating increases the temperature of the air sample by a $\Delta T_{\max }$ of $16 \mathrm{~K}$. Assuming that the air gets compressed over a distance of $\sim 0.5 \mathrm{~m}$ upstream of the instrument (cf. Wendisch and Brenguier, 2013, Sect. 6.2.1 therein), then, for the given flight velocity, the airborne particles are exposed for an overall duration of about $2 \mathrm{~ms}$ to a continuously heating environment, ending up at the $\Delta T_{\max }$ of $16 \mathrm{~K}$. The shrinkage, i.e. the evaporative loss of size, of an airborne ice particle of $2 \mu \mathrm{m}$ initial diameter is at most $\sim 5 \%$ after a $2 \mathrm{~ms}$ lasting exposure to a $\Delta T_{\max }$ of $16 \mathrm{~K}$ (at any initial air temperature of $190-245 \mathrm{~K}$ ) at a static pressure of $300 \mathrm{hPa}$, as calculated from the mass rate change (Pruppacher and Klett, 2012; Spichtinger and Gierens, 2009). The shrinkage increases vigorously for particle of initial sub-micron size. Moreover, the compression of air over a distance of $\sim 0.5 \mathrm{~m}$ upstream of the instrument causes a $\Delta p_{\max }$ of $60 \mathrm{hPa}$ (cf. Fig. 8a). If scaled to the dimensions of a droplet of millimetre-sized diameter (smaller particles are affected to lesser extent) the potential droplet deformation due to compression may be negligible.

In Fig. 9 the comparison of respectively measured $p_{2}$ is shown together with the correlation of the PAS as derived 


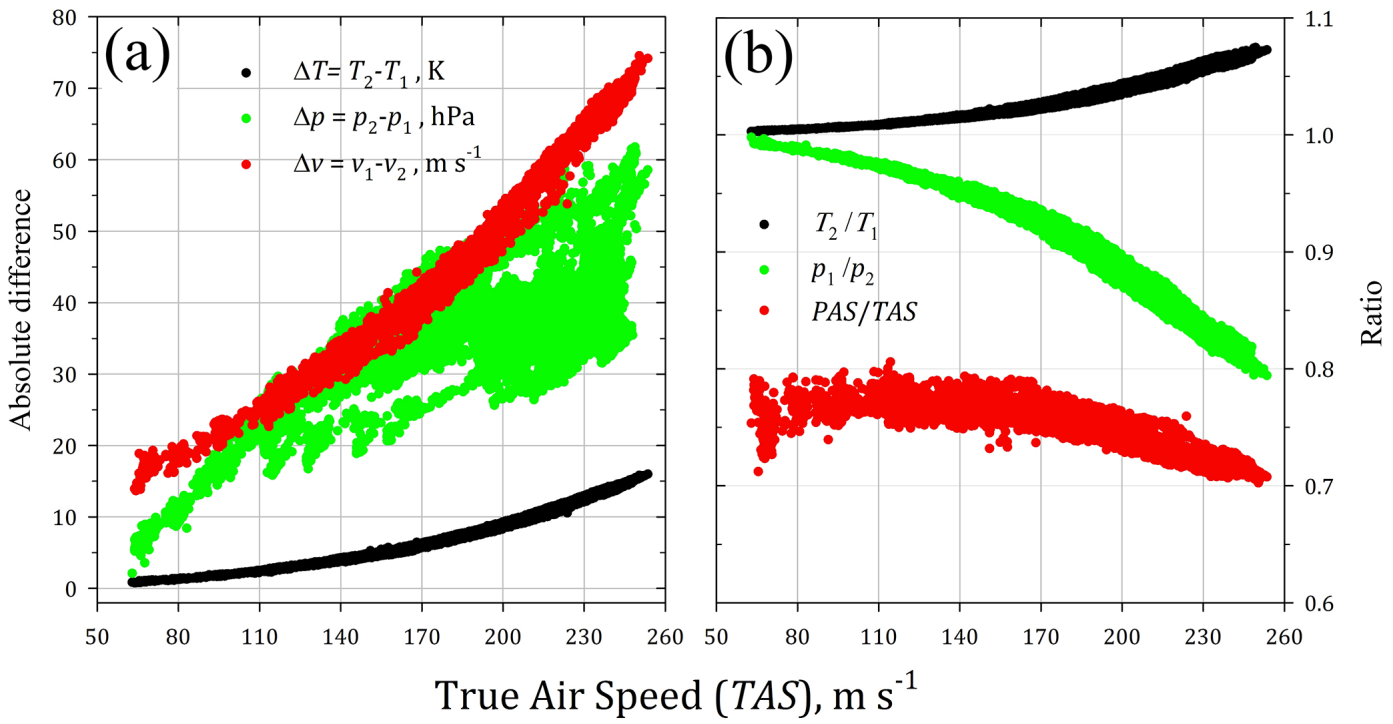

Figure 8. Relevant parameters for determining $\xi$ as a function of TAS for the CCP. (a) The absolute difference of measured pressures ( $p_{1}$, $\left.p_{2}\right)$ and velocities $\left(v_{1}, v_{2}\right)$ and the difference of measured temperature $T_{1}$ to calculated $T_{2}$. (b) The ratio of pressures $\frac{p_{1}}{p_{2}}$ and temperatures $\frac{T_{2}}{T_{1}}$ as used in Eq. (7) to illustrate respective effectiveness in the $\xi$ correction.

Table 1. Coefficients of the statistical analyses of derived $\xi$ values: (1) quadratic regression with parameters and standard deviations ( $\sigma$ ); (2) linear correlation of the instrument-specific $\xi$ values. Both the $\xi$ parameterisations and correlations from $H A L O$ measurements are based on $>130000$ single $1 \mathrm{~Hz}$ data points (>36 flight hours).

\begin{tabular}{rrrr}
\hline \multicolumn{4}{c}{ Regression for parameterisation of $\xi$ as a function of HALO-TAS: $f=y_{0}+a \cdot x+b \cdot x^{2}$} \\
\hline & CCP & NIXE-CAPS & PIP \\
\cline { 2 - 4 }$y_{0} \pm \sigma$ & $0.99 \pm 2.04 \times 10^{-4}$ & $0.99 \pm 2.36 \times 10^{-4}$ & $0.99 \pm 2.06 \times 10^{-4}$ \\
$a \pm \sigma$ & $3.18 \times 10^{-4} \pm 2.34 \times 10^{-6}$ & $2.55 \times 10^{-4} \pm 2.71 \times 10^{-6}$ & $3.10 \times 10^{-4} \pm 2.35 \times 10^{-6}$ \\
$b \pm \sigma$ & $-3.40 \times 10^{-6} \pm 6.48 \times 10^{-9}$ & $-3.30 \times 10^{-6} \pm 7.54 \times 10^{-9}$ & $-3.37 \times 10^{-6} \pm 6.54 \times 10^{-9}$ \\
$r^{2}$ & 0.99 & 0.98 & 0.98 \\
\hline \multicolumn{5}{c}{ Linear regression of instrument-specific $\xi$ inter-correlation: $f=y_{0}+a \cdot x$} \\
\hline$y_{0} \pm \sigma$ & $-0.04 \pm 2.26 \times 10^{-4}$ & PIP versus NIXE-CAPS & PIP versus CCP \\
\cline { 2 - 4 }$a \pm \sigma$ & $1.03 \pm 2.49 \times 10^{-4}$ & $-0.05 \pm 5.09 \times 10^{-4}$ & $-0.01 \pm 4.73 \times 10^{-4}$ \\
$r^{2}$ & 0.99 & $1.05 \pm 5.60 \times 10^{-4}$ & $1.01 \pm 5.20 \times 10^{-4}$ \\
\hline
\end{tabular}

from the dynamic pressure proportion of the pitot tube measurements. The correlations of the individually measured $p_{2}$ between NIXE-CAPS and CCP (Fig. 9, upper-left panel) and between PIP and CCP (Fig. 9, upper-right panel) agree almost in line with the displayed $1: 1$ relationship (dashed red lines). Thus, the $p_{2}$ measurement of the instruments does not seem to be significantly affected either by the individual probe head design or by the respective wing position. Note that the calibrated pressure transducers commonly integrated in the individual probes are of the type Honeywell, model $142 \mathrm{PC} 15 \mathrm{~A}$ with a specified linearity within $\pm 0.4 \%$ of the output signal span for the pressure range between 140 and $1030 \mathrm{hPa}$

The dynamic pressure for calculating the air speed results from the total pressure, impacting on the pitot's forwardfacing congestion tube, subtracted by the static pressure that is detected at the pitot tube's flanks. Hence, the PAS comparison between NIXE-CAPS and CCP (Fig. 9, lower-left panel) exhibits a systematic discrepancy of about 5-10 $\mathrm{m} \mathrm{s}^{-1}$ by which the resulting PAS of the CCP exceeds the NIXECAPS measurements over the entire velocity range. This may result from different calibrations of the respective pitot tube or it could be an effect of the instrument's wing position. 


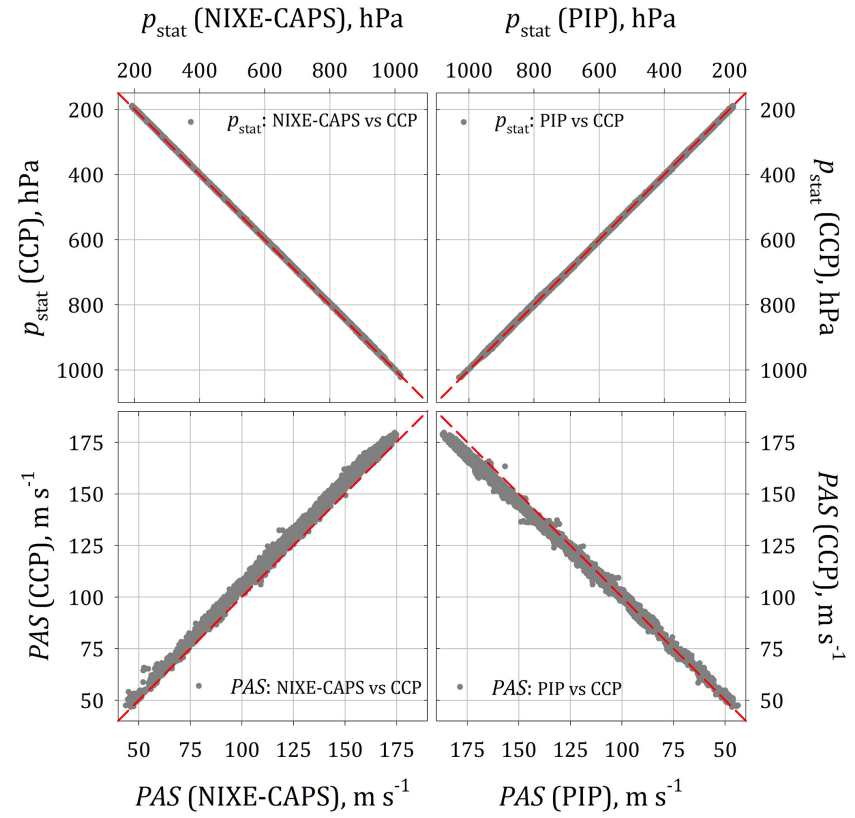

Figure 9. Correlations between individually measured static pressures and PAS for each instrument pair. The $p$ correlations (upper panels) indicate consistency as the data follow the $1: 1$ relationship (dashed red lines). The PAS correlations (lower panels) reveal systematic deviations from the $1: 1$ relationship which may be attributed to the instruments' different geometry or underwing position.

For comparably much smaller flight velocities $\left(<100 \mathrm{~m} \mathrm{~s}^{-1}\right)$, previous studies demonstrated that the air flow field changes along the wing span with different impact on instruments positioned outboard or inboard at an aircraft's wing (Drummond and MacPherson, 1985; MacPherson and Baumgardner, 1988). It is also likely that the systematically stronger deceleration of air flow upstream of NIXE-CAPS is caused by its winglet (cf. Fig. 6), which may increase the probe's cross-sectional area compared to that of the CCP. The comparison between the PIP's reconstructed PAS and the measured PAS of the CCP is also shown in Fig. 9 (lower-right panel) but the agreement among the PAS data may benefit from the implicit dependency of the dependency of the PIP's reconstructed PAS on the imported temperature data from the CCP (cf. remark above). However, a potential but unlikely deviation of the adopted temperature from a true temperature at the PIP's position by up to $\pm 20 \mathrm{~K}$ would shift resulting comparisons by at most $5 \%$ in either abscissa direction.

For providing a parameterisation of $\xi$ values as a function of TAS the data set needs to be reassessed by accounting for the limited periods of tamped or malfunctioning pitot tubes. For the following, those periods that were identified to be affected by an inoperative pitot tube have been removed from the data set. In Fig. 10 the derived $\xi$ factor is depicted as a function of TAS. The parameterisation results from fitting a quadratic regression (Table 1) to the given data set. For each

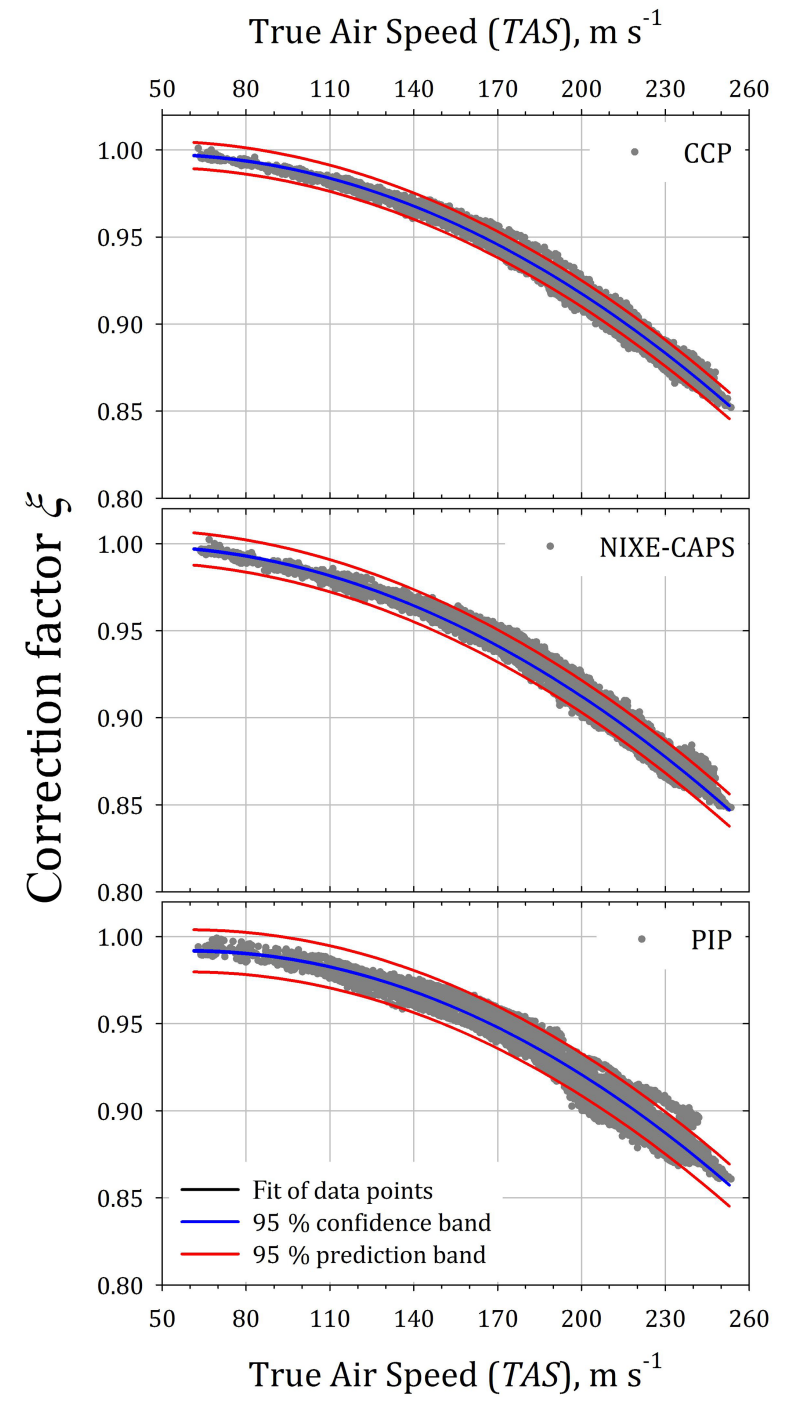

Figure 10. Parameterisation of $\xi$ as a function of TAS for the three different instruments deployed on HALO during the ML-CIRRUS mission. Parameterisation coefficients are provided in Table 1. The fit curve (black) is covered by the lines (blue) of the narrow confidence band.

instrument the individually derived parameters of $v_{2}$ (PAS) and $p_{2}$ (static air pressure at the probe) are used, such that the $\xi$ factors are also individually determined for each instrument. The regression fits in Fig. 10 are provided together with the $95 \%$ confidence band (blue lines) and the $95 \%$ prediction band (red lines). Note that the $95 \%$ confidence band is very narrow, even covering the black regression fit because the data set used for these regressions is large and the data variability is small. The fit parameters are summarised in Table 1 and the regressions always reveal values of $r^{2}$ greater than 0.98 , which confirms the solidity of the functional relationship between $\xi$ and TAS. 


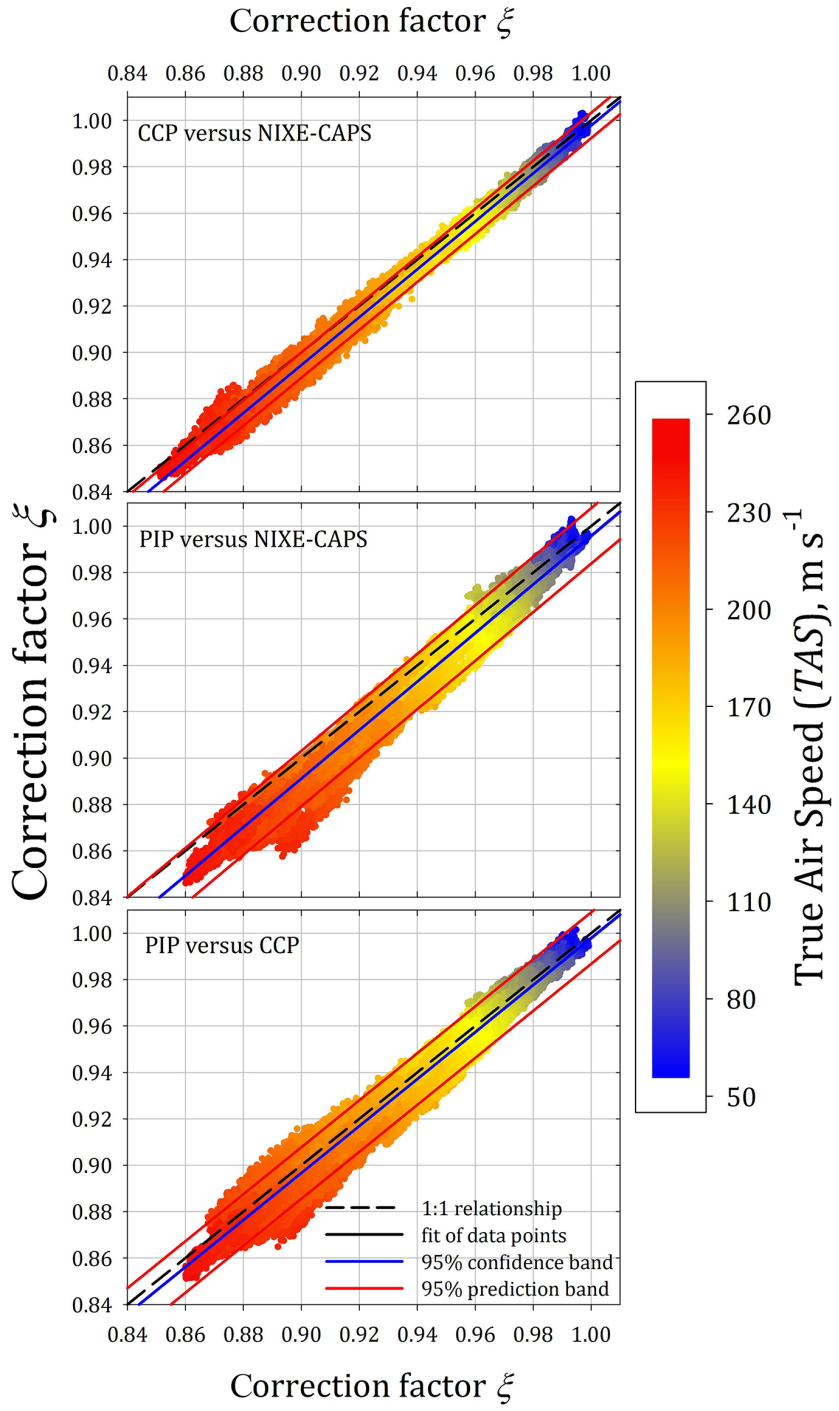

Figure 11. Correlations of instrument-specific $\xi$. The deviation from the $1: 1$ relationship (dashed black lines) as a function of the aircraft true air speed (TAS) is strongest at the PIP position (portside, innermost). Coefficients for the correlations are provided in Table 1. The fit curve (black) is covered by the lines (blue) of the narrow confidence band.

\subsection{The consistency of $\xi$ for $H A L O$ instruments}

Further insight into the properties of $\xi$ is provided by Fig. 11, which illustrates the correlation of individually derived $\xi$ values for each instrument. The $\xi$ data are colour-coded according to TAS and the linear correlation between the instrumentspecific $\xi$ data and TAS are derived. The graphs also contain the very narrow $95 \%$ confidence band (blue lines) and the $95 \%$ prediction band (red lines). The parameters for the linear correlations shown in Fig. 11 are also summarised in Table 1 . The $\xi$ values exhibit a strong correlation with a correlation coefficient $r^{2}$ larger than 0.97 , which indicates $\xi$ to be widely independent of the instrument characteristics, such as wing position or design, in contrast to $\frac{\text { PAS }}{\text { TAS }}$. Nevertheless, in detail the individual $\xi$ values obviously differ from each other, which is presumably connected to the differently shaped probe heads and/or the instrument's distance to the aircraft fuselage. Starting with neighboured instruments, i.e. comparing CCP with NIXE-CAPS (Fig. 11, upper panel) and PIP with NIXE-CAPS (Fig. 11, centred panel), an increasing deviation from the $1: 1$ relationship (dashed black lines) appears. The comparison of corresponding results from the PIP and the CCP (Fig. 11, bottom panel) depicts a smaller deviation of $\xi$ values from the 1:1 relationship. However, it must be emphasised that the dependency of the PIP's reconstructed PAS on CCP temperature data (cf. Sect. 3.2) has negligible impact on derived $\xi$. It remains speculative to ascribe the $\xi$ deviation to the individual instruments' design, although it may explain this observation.

\subsection{Effectiveness of $\xi$ corrections on atmospheric particle measurements}

In the following the derived $\xi$ values are applied to data of atmospheric cloud measurements that were performed during the HALO mission ACRIDICON-CHUVA. The studies of various types of tropical convective cells aimed at, amongst other characteristics, the microphysical properties of cloud elements under variable conditions. Large contiguous cloud fields with liquid or mixed-phase cloud particles were probed, occasionally over more than $30 \mathrm{~min}$ without encountering cloud-free air. Relatively high particle number concentrations were detected. For demonstrating the effectiveness of the $\xi$ correction a segment was selected from flight "AC13" on 19 September 2014 between 20:00:40 UTC (72 $040 \mathrm{~s}$ of day) and 20:32:00 UTC (73920 s of day). During this flight period, at almost constant level flight at about $13 \mathrm{~km}$ altitude (cf. Table 2), spheroidal particles were mostly present and detected as such. Figure 12 shows a time series (left panel) of the total number concentration (as $10 \mathrm{~s}$ running averages) derived from measurements of both the CCP (CDP and CIPgs) and the PIP. Additionally, a particle size distribution covering the full diameter detection range of CCP and PIP is provided (Fig. 12, right panel) averaged over the complete level flight period to reduce the counting error level to a minimum over the full diameter range covered. In both graphics, the time series and according particle size distribution are shown in four ways:

1. measured particle number concentration $N_{\text {meas }}$ (black);

2. the data corrected by values of $\xi$ determined for each second of measurement (green);

3. the data corrected by values of $\xi$ (as before) and $\mu\left(D_{\mathrm{p}}\right)$ (cf. Sect. 2.5) (blue);

4. the data set after correction with $1 \mathrm{~Hz}$ resolved factors of $\frac{\text { PAS }}{\text { TAS }}$ (red). 

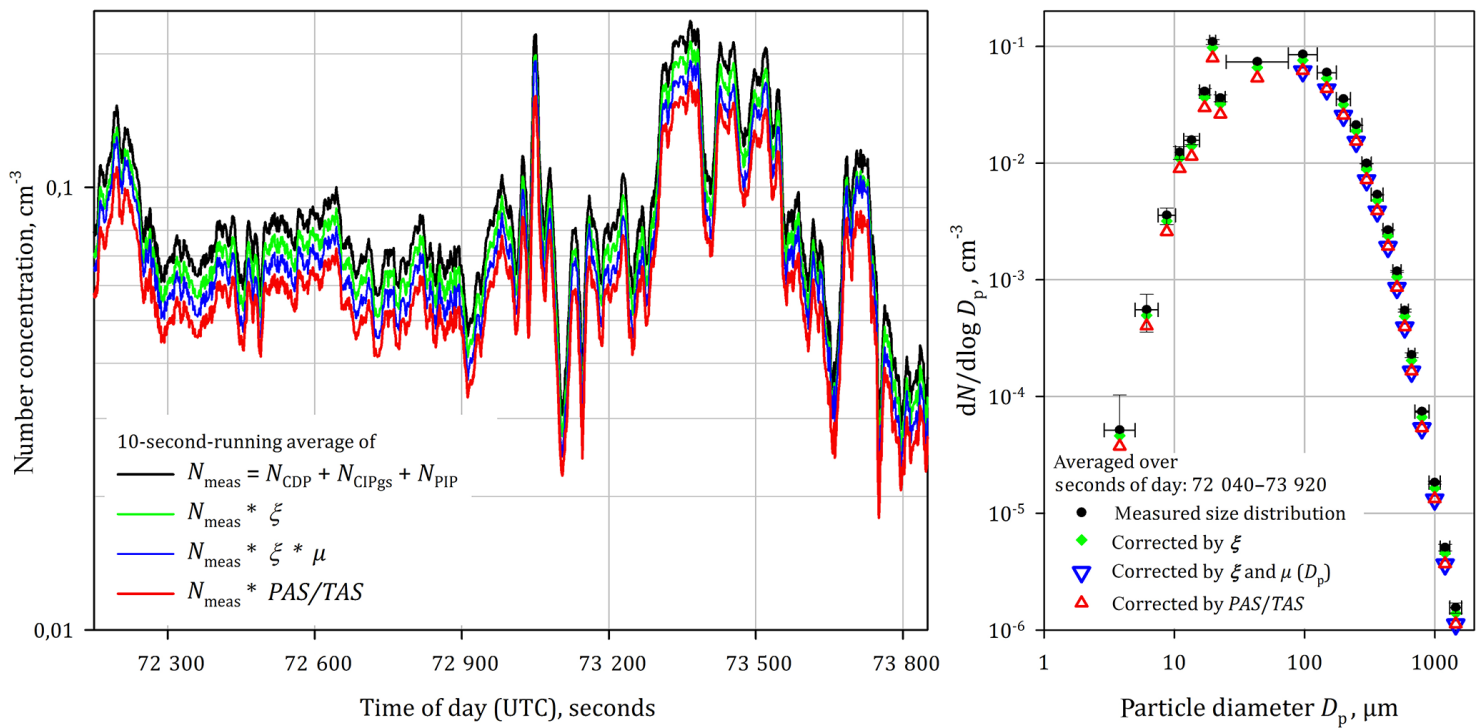

Figure 12. Resulting particle number concentration after application of different correction procedures to the data acquired during the ACRIDICON-CHUVA mission flight "AC13" on 19 September 2014 between 20:00:40 UTC (72 040 s of day) and 20:32:00 UTC (73 920 s of day), over the Amazonian basin, Brazil. Left panel: the time series of total particle concentration measured with CCP and PIP. Right panel: the resulting particle size distribution, merged from CCP (CDP and CIPgs) and PIP measurements. As a consequence of used binning scheme of OAP data the inertia correction with $\mu$ is applied to the particle size bin of $D_{\mathrm{p}}=97 \pm 25 \mu \mathrm{m}$ and to all larger sizes $(\mu=0.8$, cf. Eq. 9).

Table 2. Altitude and flight velocity for the ACRIDICON-CHUVA mission flight "AC13" on 19 September 2014 over the time period between 20:00:40 UTC (72 040 s of day) and 20:32:00 UTC (73920s of day) and derived corrections of $\xi$ and $\frac{\text { PAS }}{\text { TAS }}$ for CCP and PIP. Inertia correction is applied with $\mu$ for particles with $D_{\mathrm{p}}>96 \mu \mathrm{m}$.

\begin{tabular}{llllllll}
\hline & $\begin{array}{l}\text { Flight altitude } \\
\text { in m (a.s.l.) }\end{array}$ & $\begin{array}{l}\text { TAS } \\
\text { in } \mathrm{m} \mathrm{s}^{-1}\end{array}$ & $\xi-\mathrm{CCP}$ & $\xi-\mathrm{PIP}$ & $\mu\left(D_{\mathrm{p}}\right)$ & $\frac{\text { PAS }}{\text { TAS }}$ CCP & $\frac{\text { PAS }}{\text { TAS }}$ PIP \\
\hline Average & 12971.6 & 221.75 & 0.90 & 0.89 & 0.8 & 0.73 & 0.76 \\
Maximum & 12980.7 & 226.89 & 0.91 & 0.89 & 0.8 & 0.74 & 0.77 \\
Minimum & 12962.5 & 215.12 & 0.89 & 0.88 & 0.8 & 0.72 & 0.74 \\
\hline
\end{tabular}

The averaged TAS $\left(\sim 220 \mathrm{~m} \mathrm{~s}^{-1}\right.$, cf. Table 2$)$ over the depicted time period suggests a $\xi$ value that causes an effective correction of $N_{\text {meas }}$ of about $10 \%$, read off Fig. 10. The averaged values of calculated $\xi$ for the CCP and PIP are indeed close to 0.9 (cf. Table 2). The additional correction with $\mu\left(D_{\mathrm{p}}\right)$ affects the particle number concentrations in the spectral size bin of $D_{\mathrm{p}}=97 \pm 25 \mu \mathrm{m}$ and those of larger sizes. However, a correction with $\frac{\text { PAS }}{\text { TAS }}$ would cause a downscaling of $N_{\text {meas }}$ by up to $27 \%$ (for the CCP-detected particle size range, cf. Table 2) whereas the applied $\xi$ corrects $N_{\text {meas }}$ with regard to the air's compression systematically by about $10-11 \%$. Taking the droplets' inertia into account, the applied correction by $\xi$ and $\mu$ (in total by about $13 \%$ ) effects a comparatively cautious change with respect to $N_{\text {meas }}$ when predominantly smaller particles are encountered (e.g. at the UTC times $72110,73050,73140$ or the period between 73070 and $73700 \mathrm{~s}$ of the day). In periods when par- ticles with sizes $D_{\mathrm{p}}>100 \mu \mathrm{m}$ prevail the correction by $\xi$ and $\mu$ effectively depletes $N_{\text {meas }}$ by at the most $21 \%$.

In essence, the effective correction of $N_{\text {meas }}$ by $\xi$ may not be excessive but, by knowing the variables to determine $\xi$, a systematic bias in measured particle number concentrations is easily eliminated. The additional correction by $\mu\left(D_{\mathrm{p}}\right)$ allows for further approximation of $N_{\mathrm{amb}}$ out of $N_{\text {meas }}$. However, it needs to be noted that $\mu$ is of varying effectiveness, depending on the particle size and on the cloud particles' phase. The correction of $N_{\text {meas }}$ by $\xi$ and $\mu$ finally becomes very effective when surface, volume or mass distributions are obtained from the OAP measurements. Furthermore, if, for example, the cloud's liquid water content (LWC) or ice water content (IWC) is extracted from OAP data, the compression correction in combination with a carefully chosen inertia correction over the entire range of particle sizes detected is essential to base any conclusion on reasonable data. 
Table 3. Coefficients of the statistical analyses of derived $\xi$ values for CCP measurements on the Learjet-35A: quadratic regression with parameters and standard deviations $(\sigma)$. The $\xi$ parameterisation is based on $>12000$ single $1 \mathrm{~Hz}$ data points ( $\approx 3.3$ flight hours).

\begin{tabular}{rr}
\hline \multicolumn{2}{l}{$\begin{array}{l}\text { Regression for parameterisation of } \xi \\
\text { as a function of Learjet-TAS: cf. Table } 1\end{array}$} \\
\hline \multicolumn{1}{c}{ CCP } \\
\hline$y_{0} \pm \sigma$ & $0.99 \pm 6.91 \times 10^{-4}$ \\
$a \pm \sigma$ & $3.74 \times 10^{-4} \pm 8.35 \times 10^{-6}$ \\
$b \pm \sigma$ & $-3.08 \times 10^{-6} \pm 2.48 \times 10^{-8}$ \\
$r^{2}$ & 0.97 \\
\hline
\end{tabular}

\subsection{Correction factor $\xi$ for other fast-flying aircraft}

Provided that $\xi$ was sufficiently proven to hold for large ranges of atmospheric conditions and aircraft speeds, the question arises whether the properties of $\xi$ can also hold for other fast-flying aircraft.

In Fig. 13a the determined $\xi$ values for each time of $\mathrm{CCP}$ measurement on board the Learjet-35A are displayed from a single flight (over 3.3 flight hours) on 5 September 2013 during the AIRTOSS-ICE (AIRcraft TOwed Sensor Shuttle) mission aiming at ice clouds (cirrus) over northern Germany (Finger et al., 2016). The $\xi$ values for one flight, when CCP was deployed on an underwing position of the Learjet$35 \mathrm{~A}$, are parameterised by means of a quadratic regression and according coefficients are summarised in Table 3. The derived $\xi$ values (green dots) as a function of TAS follow in general the expected course and the data set is similarly compact over the TAS range as previously shown for the $H A L O$ cases. However, the comparison of the $\xi$ fit from the Learjet-35A measurements with the parameterised $\xi$ from $H A L O$ flights (dark cyan line) reveals that $\xi$ for the Learjet$35 \mathrm{~A}$ configuration generally causes a smaller correction of $N_{\text {meas }}$ to reach $N_{\mathrm{amb}}$. As the instruments deployed on board of HALO and the Learjet-35A are identical the systematic difference in $\xi$ indicates that the compression may depend on the instrument-platform configuration. As $\xi$ is principally a measure for the compression strength, it seems that upstream of the CCP on the Learjet-35A, for some reason, the air compression is weaker than on $H A L O$. Factually, the instrument configuration by using the AIRTOSS, which is released from the Learjet-35A on a steel cable during flight (Frey et al., 2009), displaces the CCP measurements to a certain distance (up to $4000 \mathrm{~m}$ ) away from any potential source of disturbance or interference provided by the aircraft. The data sets of two flights of the AIRTOSS-ICE campaign were selected which provide all variables required to determine $\xi$. Measurements during curved manoeuvres were discarded from further analysis as the adaptation of the AIRTOSS's flight attitude to rough changes in flight direction is delayed. Moreover, the CCP pressure data were adjusted to account for the differ- ence of static air pressure accompanied with the lower flight altitude of AIRTOSS with respect to the Learjet-35A. For the levitating CCP the calculated $\xi$ values are displayed in Fig. 13b (purple dots) exhibiting a strong scatter. However, hereby a dispersion of $\xi$ about a mean appears to be indicated that is close to the $\xi$ parameterisation fit of measurements with CCP when attached under the Learjet's wing. Thus, a significant influence of the aircraft's wings or the fuselage of the Learjet-35A on the underwing-mounted CCP is not definitely verifiable. Concerning the difference of $\xi$ resulting from measurements either on the Learjet-35A or the $G-550$ $H A L O$ we can only surmise that a specific flow field is induced due to the specific $H A L O$ configuration, by the aircraft fuselage and/or the wings' leading edge and/or the pairwise configuration of the underwing instruments. Nevertheless, by applying $\xi$ the individual and systematic influences on the actual thermodynamic conditions under which the measurements occur are, to a large extent, accounted for - independent of the probably various and likely interfering sources of disturbances on the measurement conditions.

\section{Summary and conclusions}

To determine particle number concentrations with respect to ambient conditions from the measurements of underwing cloud probes on fast-flying aircraft two corrections need to be applied on the data obtained under measurement conditions from the free atmosphere.

The first correction refers to the compression of air occurring upstream of the air flow obstacle provided by the instrument's body. Taking the compression effect into consideration is of increasing importance with increasing flight speeds (e.g. up to $250 \mathrm{~m} \mathrm{~s}^{-1}$ ). This first correction accounts for the compressed state of the sample air volume probed per time interval as indicated by an observable increase of the static air pressure (and air temperature) in the instruments' detection region compared to ambient conditions.

The particles' mobility to react on small-scale and fast changes in a flow field, inducing a particle's displacement from its initial state, is a function of the particles' individual mass (i.e. inertia). Thus, the second correction refers to the particle inertia which impacts the penetration speed of cloud particles while passing the instrument's detection region. Due to the compression of air the flow in the instruments' detection region is decelerated compared to the undisturbed state (at a certain distance away from any obstacle) to which large cloud droplets (e.g. $\left.D_{\mathrm{p}}>100 \mu \mathrm{m}\right)$ are unable to adapt fast enough. However, strong indications were found that the penetration speed of smallest particles (e.g. $\left.D_{\mathrm{p}}<70 \mu \mathrm{m}\right)$ is more than likely affected by the changed air flow conditions. As a consequence of the particles' individual inertia, the penetration speed through the instruments' detection region is not equal for all particles in a cloud. Thus, the general assumption of the constancy of particle mixing 
ratios (particle number per mass of air sampled) is not fulfilled over the entire cloud droplet size range. Indeed, the mixing ratio between ambient and measurement conditions likely remains constant only for small-sized particles which exhibit enough mobility to adapt to the air compression. In contrast, large particles may accumulate in the instruments' detection region due to their inability to kinetically adapt to the changing air speed. Hence, without an inertia correction of measured particle number concentrations, large particles may be over-represented in the sampled air volume.

For the compression correction the factor $\xi$ is introduced based on thermodynamic considerations for inverting the data from the pressure and temperature conditions during measurement to ambient conditions. An equation is provided for deriving $\xi$ that depends on the variables of static pressure and temperature in the ambient (undisturbed) state as well as the aircraft TAS. Additionally, the static pressure measurement and the actually measured PAS at the individual probe are needed. Provided that an underwing probe is equipped with a pitot tube for continuous measurement of air speed and static pressure in the vicinity of the probe, the instrumentspecific $\xi$ can be derived for each second of measurement during a flight.

As the detector scanning of OAPs is triggered by the measured airflow velocity, a significant deviation or falsification of the pitot-measured PAS would result in visibly distorted images. Measurement flight sections featuring predominantly liquid (spherical) cloud droplets were selected. For these chosen measurement periods the grade of distortion of the recorded images was apportioned to the deviation of the particle penetration speed through the instrument's detection region by means of an automated OAP image analysis. Small particles of $D_{\mathrm{p}}<70 \mu \mathrm{m}$ appear to largely adapt to the decelerated air speed by reaching $v_{\mathrm{p}} \approx \mathrm{PAS}$ in the instruments' detection region. Larger droplets with $D_{\mathrm{p}}>100 \mu \mathrm{m}$ seem to penetrate the detection plane with PAS $<v_{\mathrm{p}}<$ TAS, which is attributable to the particles' inertia. The dependence of the inertia correction on the particle size is expressed in the suggested correction factor $\mu\left(D_{\mathrm{p}}\right)$ to be applied to measured particle number concentration $N_{\text {meas }}$. For the particular case investigated the correction of measured number concentrations with $\mu=1$ holds for cloud droplets of $D_{\mathrm{p}}<70 \mu \mathrm{m}$ while a correction with $\mu=0.8$ seems to suffice for cloud droplets with $100 \mu \mathrm{m}<D_{\mathrm{p}}<225 \mu \mathrm{m}$. For larger droplets (e.g. $D_{\mathrm{p}}>225 \mu \mathrm{m}$, which were not detected during investigated measurement periods) the inertia correction may asymptotically approach a value of $\mu=0.75$. Only if the particles' inertia inhibits a change of the particles' initial state may the resulting particle penetration speed through the OAP detection region satisfy the assumption that $v_{\mathrm{p}} \approx \mathrm{TAS}$. However, the generalized correction regarding the particle's inertia based on the particle's size may not always be appropriate. Prior to applying such a correction some careful considerations are required regarding the particles' phase and habit. Cloud particles exhibit the largest material density when they are liq- uid, whereas, if a cloud particle is frozen, its material density (and inertia) is decreased compared to a liquid droplet of the same geometrical size. Moreover, spatial structures of ice particles (e.g. if evolved to dendrites, crystals, bullet rosettes or hollow needles) may lead to increased mobility due to a decreased compactness of the particles.

At present there are three different approaches to treat particle microphysical data obtained from underwing probes.

1. Without knowledge of the air speed and static pressure at the point of measurement it is common practice to presume that particles always penetrate the probe's sample area $A_{\mathrm{S}}$ with speeds equal to the aircraft TAS. Taking this route, the compression of air due to the moving instrument body and the compression-induced motion of particles out of their ambient (undisturbed) state is ignored. As a consequence of the compression upstream of a probe, the PAS at the point of measurement must be systematically lower compared to TAS. Determining particle number concentrations by using the TAS without adjustments regarding the compression leads to an underrepresentation of particle concentrations that is not negligible.

2. The measured particle number concentration $N_{\text {meas }}$ (based on the recorded PAS; cf. Sect. 2.1 for details) does not represent the ambient number concentration of cloud particles. The compression of air causes a modification of the particle's environment and behaviour at the point of measurement compared to ambient (undisturbed) conditions. Thus, the measured particle number concentrations without any corrections may be representative for the measurement conditions only. However, compared to ambient particle number concentrations, the uncorrected $N_{\text {meas }}$ is an overestimate of increasing strength with flight velocity. Note that an uncertainty of $N_{\text {meas }}$ remains due to the PAS uncertainty which may not considerably exceed $10 \%$.

3. Multiplying measured particle number concentrations $N_{\text {meas }}$ with the ratio $\frac{\mathrm{PAS}}{\mathrm{TAS}}$, presumably deduced from geometric considerations, with the attempt to hereby invert the measured concentrations to ambient conditions lacks any physical rationale. The ratio of air speeds does not account for the compression of air upstream of the probe which is the major reason for the deviation of measured air speeds, PAS and TAS. By using the ratio $\frac{\text { PAS }}{\text { TAS }}$, the increase of pressure and the heating accompanied with the compression of air remains fully ignored. Hence, this simplified correction procedure turns out to cause an unreasonable reduction of particle number concentrations. This procedure was shown to affect the results at surprisingly low aircraft speeds.

Therefore, the particle number concentration under measurement conditions $N_{\text {meas }}$ (based on the recorded PAS, cf. 
Sect. 2.1) needs correction to account for the compression of air and the compression-induced motion of particles out of their ambient (undisturbed) state, which is a function of the cloud particles' individual mass (i.e. inertia). The herein introduced correction factor $\xi$ covers the most important impacts accompanied by the compression. It is suggested that the subsequent correction of the measured particle number concentration with the factor $\mu\left(D_{\mathrm{p}}\right)$ to account for the particles' individual inertia be applied carefully. Further effects may be considered which additionally concern the ability of the cloud particles to adapt to sudden changes of the flow field upstream of an underwing probe. CFD simulations by Korolev et al. (2013), for example, indicate that the air speed locally varies along the sample volume. Thus, the complexity of velocity corrections increases when an inhomogeneity of the air speed distribution within the sample volume is also taken into consideration.

If pitot tube measurements are not available, the provided $\xi$ parameterisation as a function of TAS serves as a guideline for adaptation of the TAS to determine particle number concentrations. The parameterisation also shows that the compression effect is comparatively small for lower flight velocities. For a mean cruising speed, e.g. of the M-55 Geophysica of about $170 \mathrm{~m} \mathrm{~s}^{-1}$, the systematic bias of the number concentration obtained from CCP measurements may be at most $6 \%$ in the case that the compression is not otherwise accounted for and if the measured number concentrations are directly determined by using the recorded PAS. Note that this potential bias of CCP measurements on board the $M-55 \mathrm{Geo}$ physica is directly taken from the parameterisation of $\xi$ for the CCP on HALO (cf. Fig. 10) and therefore represents the uppermost extreme. For slower aircraft, e.g. the POLAR 5 (a modified and turboprop-engined Douglas DC-3) with cruising speed of about $70 \mathrm{~m} \mathrm{~s}^{-1}$ (Klingebiel et al., 2015) the potential bias of uncorrected CCP-measured particle number concentrations is at worst $2 \%$ and therefore lies well within the measurement uncertainty. Calculated $\xi$ from CCP measurements on board the Learjet-35A, reaching flight velocities comparable to those of the G-550 HALO, reveals that the compression is generally less expressed in the Learjet$35 A$ configuration compared to that of the G-550 HALO. An increased compression effect on the G-550 HALO is not unambiguously connectable to a specific source. It can only be surmised that on the G-550 HALO a strengthened disturbance on the thermodynamic conditions of underwing probe measurements is accompanied with interferences of the aircraft fuselage and/or the wings' leading edge and/or the pairwise configuration of the underwing instruments.

This study provides a starting point for further intensive instrumental comparisons and investigation by means of combined measurement results of cloud and aerosol probes from the accomplished $H A L O$ field missions ML-CIRRUS and ACRIDICON-CHUVA. However, to make the measurements comparable, a common standard of treating the data and of considering systematic influences on the measurement is es-

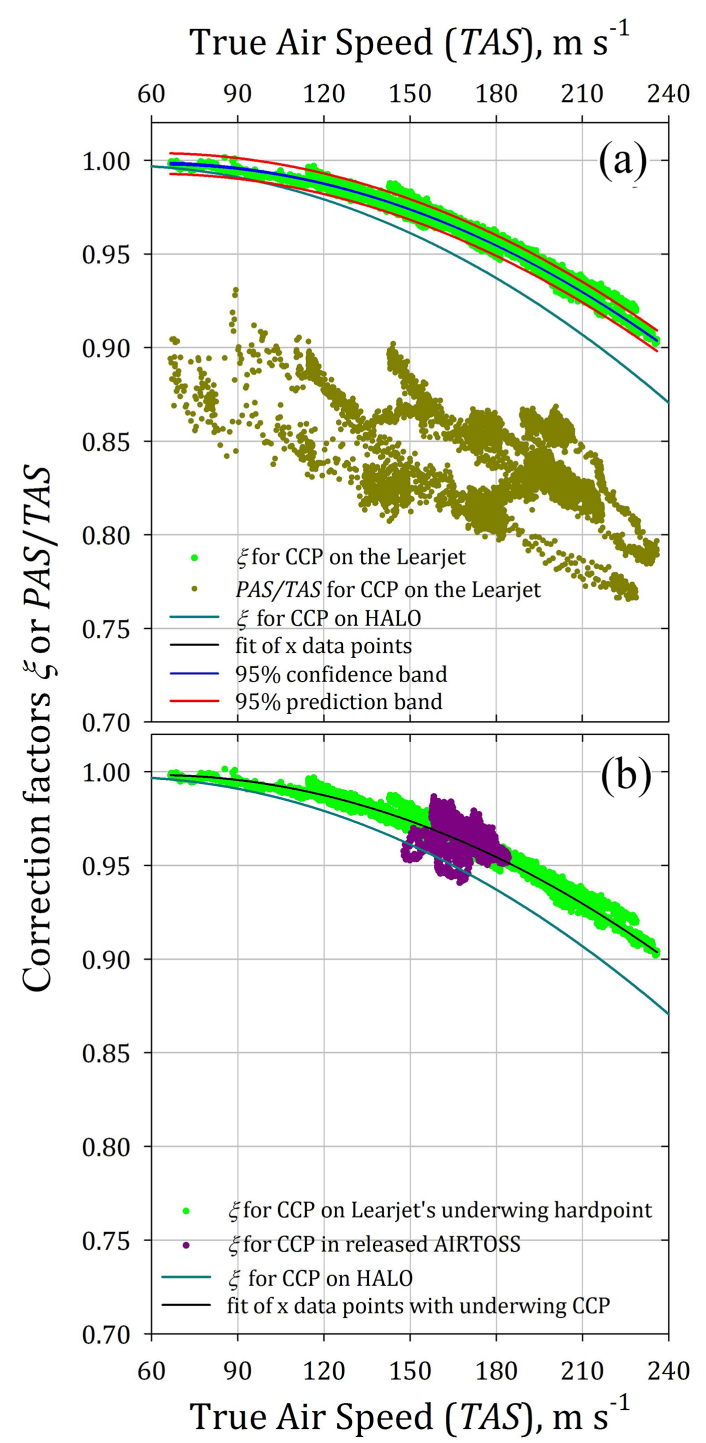

Figure 13. Comparison of corrections for the CCP (a) with the CCP attached to a Learjet-35A' underwing hardpoint (flight on 5 September 2013) during the AIRTOSS-ICE mission over northern Germany. The $\frac{\text { PAS }}{\text { TAS }}$ correction exhibits broad scatter and ambiguities. Instead, the determined $\xi$ values yield compactness over the complete TAS range. For comparison the $\xi$ parameterisation from HALO measurements of the CCP is implied, illustrating the dependence of $\xi$ on the used measurement platform. (b) Instead of $\frac{\text { PAS }}{T A S}$, the $\xi$ values are shown for the CCP when deployed in the AIRcraft TOwed Sensor Shuttle (AIRTOSS) released from the Learjet-35A on a steel cable.

sential. This standard needs to be designed and agreed upon before comparing or interpreting the data. The introduced correction procedures may serve as one contributing factor accounting for the most significant impacts resulting from the moving instrument body in the medium air on fast-flying aircraft. Further flow disturbances due to aircraft components (e.g. the impact of turbulence along the wings or the flow im- 
pacts induced by the fuselage) are potentially not covered by suggested procedures but may be subject of detailed CFD simulations.

\section{Data availability}

The meteorological and avionic data and the data products of the cloud probe measurements on board $H A L O$ for both field missions, ML-CIRRUS and ACRIDICON, are available at https://halo-db.pa.op.dlr.de/, maintained by the German Aerospace Center (DLR), Institute of Atmospheric Physics, Oberpfaffenhofen, Germany.

Instruments' basic data (i.e. pressure, temperature, PAS) as well as raw image files are stored on individual databases and are accessible upon request towards the operators of respective instrument (i.e. NIXE-CAPS: IEK-7: Stratosphere, Water Vapor and Clouds, Forschungszentrum Jülich; CCP; and PIP: Particle Chemistry Department, Max Planck Institute for Chemistry, Mainz).

Data products from measurements on board the Learjet$35 \mathrm{~A}$ are accessible upon request to the operator of respective instrument (CCP: Particle Chemistry Department, Max Planck Institute for Chemistry, Mainz). Meteorological measurements and avionic data belonging to the Learjet-35A field mission AIRTOSS-ICE were provided by enviscope GmbH, Frankfurt, Germany. 


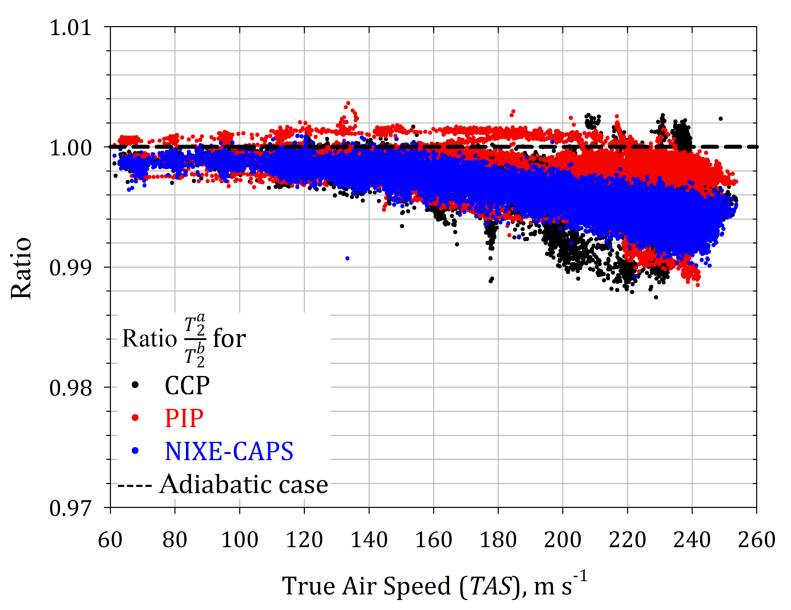

Figure A1. Assuming $T_{2}^{b}$ as the reference, for which potentially involved diabatic processes remain considered, the ratio $\frac{T_{2}^{a}}{T_{2}^{b}}$ indicates the strength of deviation from this reference. The ratio $\frac{T_{2}^{a}}{T_{2}^{b}}$ overall remains between 0.988 and 1.004 and the majority of data range at 0.99-1.0. Thus changes of state accompanied with compression are almost entirely adiabatic. The ratio $\frac{T_{2}^{a}}{T_{2}^{b}}$ deviates from the adiabatic threshold (black line) by generally less than $1 \%$, and the deviation increases expectedly with TAS potentially due to turbulence and accompanied mixing.

\section{Appendix A: Various ways of deriving the $\xi$ correction}

The derivation of $\xi$ emanates from following different conditions, which are illustrated in Fig. 1 for the air velocity $v$, the air pressure $p$, the specific enthalpy $h$ of a uniform system and the gravitational potential $\phi$.

- Condition 1 - at the aircraft's air data boom: $v_{1}, p_{1}, T_{1}$, $h_{1}, \phi_{1}$.

- Condition 2 - upstream of the probe: $v_{2}, p_{2}, T_{2}, h_{2}, \phi_{2}$.

Bernoulli's law for compressible gases and under the presumption of energy conservation reads as

$\frac{1}{2} v_{1}^{2}+h_{1}=\frac{1}{2} v_{2}^{2}+h_{2}$

assuming that the gravitation potential $\phi_{1}=\phi_{2}$ since the relative elevation between the air data boom and the underwing probe position is negligibly small, i.e. $<10 \mathrm{~m}$.

For finite differences of the specific enthalpy $\left(\Delta h=h_{2}-\right.$ $h_{1}$ ) for an ideal gas such as the air, one can use

$\Delta h=c_{\mathrm{p}} \Delta T$.

wherein $c_{\mathrm{p}}$ denotes the specific heat capacity of dry air (in $\mathrm{J} \mathrm{kg}^{-1} \mathrm{~K}^{-1}$ ) at a constant pressure.

For the further derivation we assume the following:

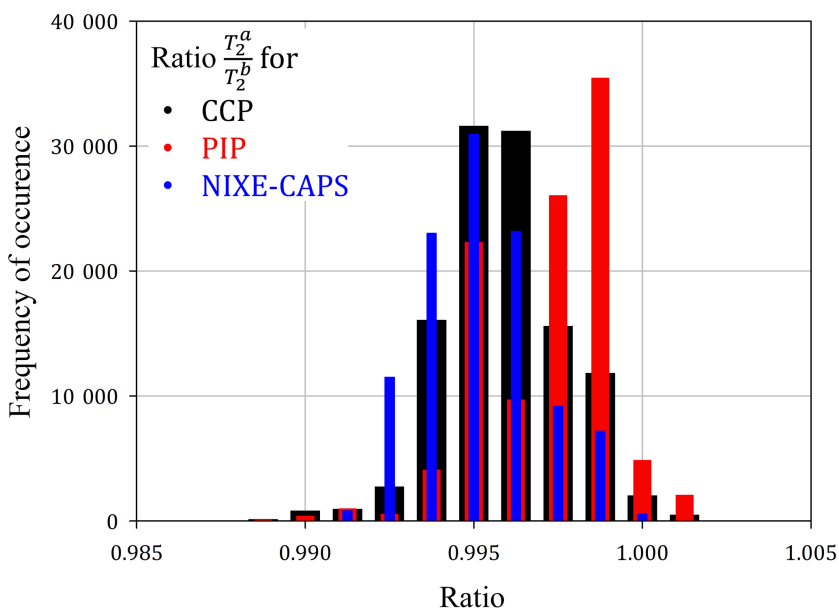

Figure A2. The occurrence frequency of $\frac{T_{2}^{a}}{T_{2}^{b}}$ values in the data set displayed in Fig. A1.

1. The pressures $p_{1}$ (static air pressure) and $p_{2}$ (static air pressure at the probe during measurement) are measured with sufficient certainty.

2. For velocities relative to undisturbed ambient cloud conditions the velocity $v_{1}$ equals the avionic TAS while $v_{2}$ is the air speed determined from the probe's pitot measurements, PAS.

3. Under undisturbed ambient conditions, for which $p_{1}$ and $T_{1}$ are valid, the particles' initial velocity relative to the aircraft flight direction may be close to zero, or at least much smaller than $v_{1}$ and $v_{2}$.

Subsequently, Eq. (A1) leads to

$\frac{1}{2}\left(v_{1}^{2}-v_{2}^{2}\right)=h_{2}-h_{1}=c_{\mathrm{p}} \Delta T=c_{\mathrm{p}}\left(T_{2}-T_{1}\right)$.

Hence, rather than the velocity ratio (cf. Sect. 2.2), the difference of the squared velocities appears in the thermodynamic approach. With Eq. (A3) the functional relationship between the aircraft air speed, reduced by the compressioninduced airflow velocity during measurement, i.e. the expression $v_{1}^{2}-v_{2}^{2}$, is provided versus the relative heating of the probed air with respect to ambient conditions. Consequently, the resulting squared velocity difference implies the change of the particles' motion in-line with the flight direction due to the compression.

Rearrangement of Eq. (A3) leads to

$T_{2}=T_{1}+\Delta T=T_{1}+\frac{1}{2 \cdot c_{p}}\left(v_{1}^{2}-v_{2}^{2}\right)$.

The specific heat capacity $c_{p}$ of air ranges from about 1002.5 to $1006.4 \mathrm{~J} \mathrm{~kg}^{-1} \mathrm{~K}^{-1}$ for atmospheric temperature conditions between 180 and $325 \mathrm{~K}$ (Dixon, 2007). Accepting an implied 


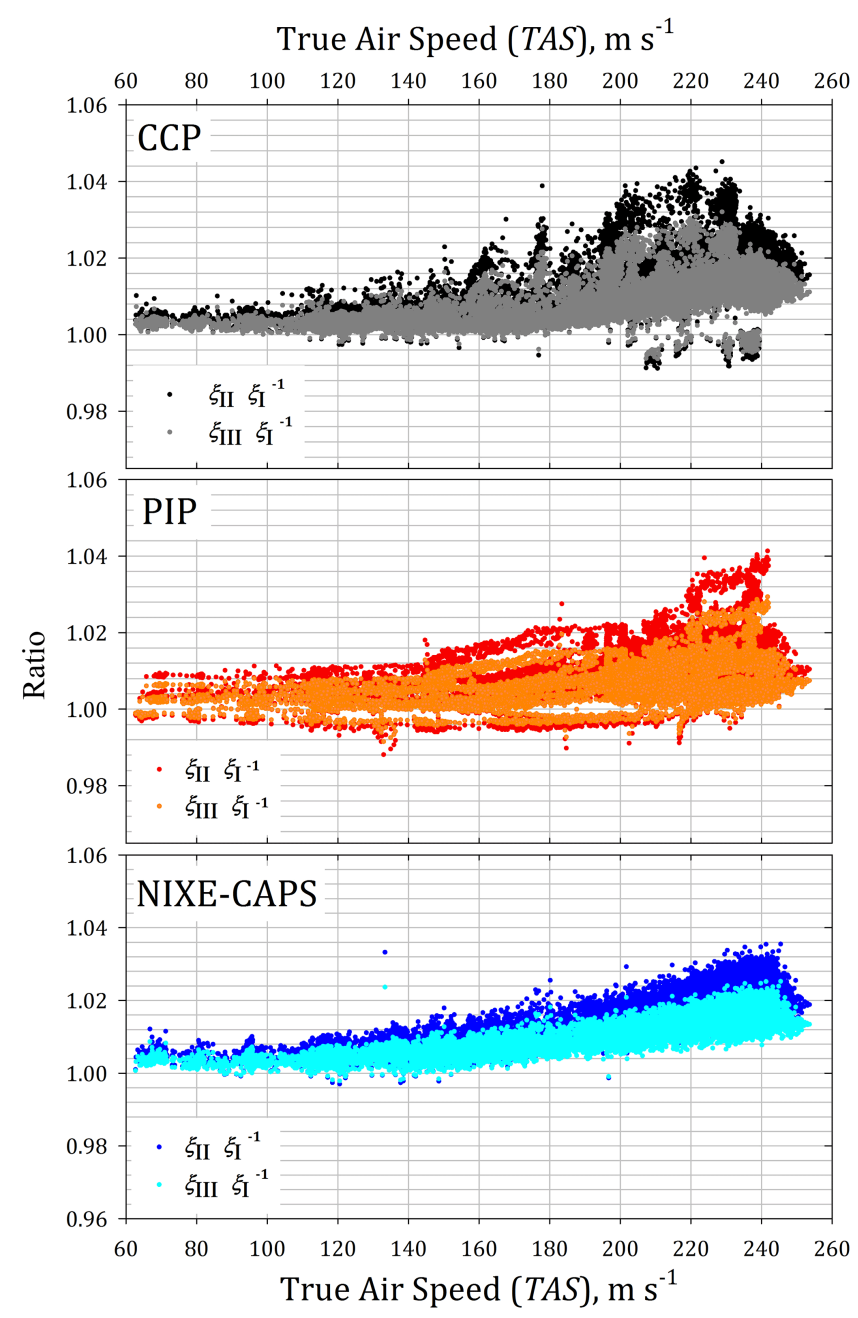

Figure A3. The ratios $\frac{\xi_{\text {II }}}{\xi_{I}}$ and $\frac{\xi_{\text {III }}}{\xi_{I}}$ as a function of TAS for the different underwing probes CIP, PIP and NIXE-CAPS, respectively. The ratios overall remain between 0.985 and 1.045 indicating a strong agreement between the differently derived $\xi$ values. The comparison generally exhibits a relative deviation of less than $5 \%$ and, over a large range of TAS $\left(<190 \mathrm{~m} \mathrm{~s}^{-1}\right)$, of even less than $2 \%$. For all instruments the correction factors $\xi_{\text {I }}$ and $\xi_{\text {III }}$ show best agreement.

uncertainty in the per-mill range, the product $2 \cdot c_{\mathrm{p}}$ in Eq. (A4) may be replaced by $2008 \mathrm{~J} \mathrm{~kg}^{-1} \mathrm{~K}^{-1}$.

Substitution of $T_{2}$ from Eq. (A4) into Eq. (5) leads to the thermodynamic correction of measured particle number concentrations to account for the compression of air upstream of the probe during flight:

$$
\begin{aligned}
N_{\mathrm{amb}} & =N_{\text {meas }} \cdot \frac{p_{1}}{p_{2}} \cdot\left(1+\frac{1}{2008 \mathrm{~J} \mathrm{~kg}^{-1} \mathrm{~K}^{-1} \cdot T_{1}}\left(v_{1}^{2}-v_{2}^{2}\right)\right) \\
& =N_{\text {meas }} \cdot \xi .
\end{aligned}
$$

By means of Eq. (A5) the thermodynamic correction factor $\xi$ is introduced, which basically equals the ratio of the probed volume and according ambient volume $\left(\frac{V_{2}}{V_{1}}\right)$ of air and which

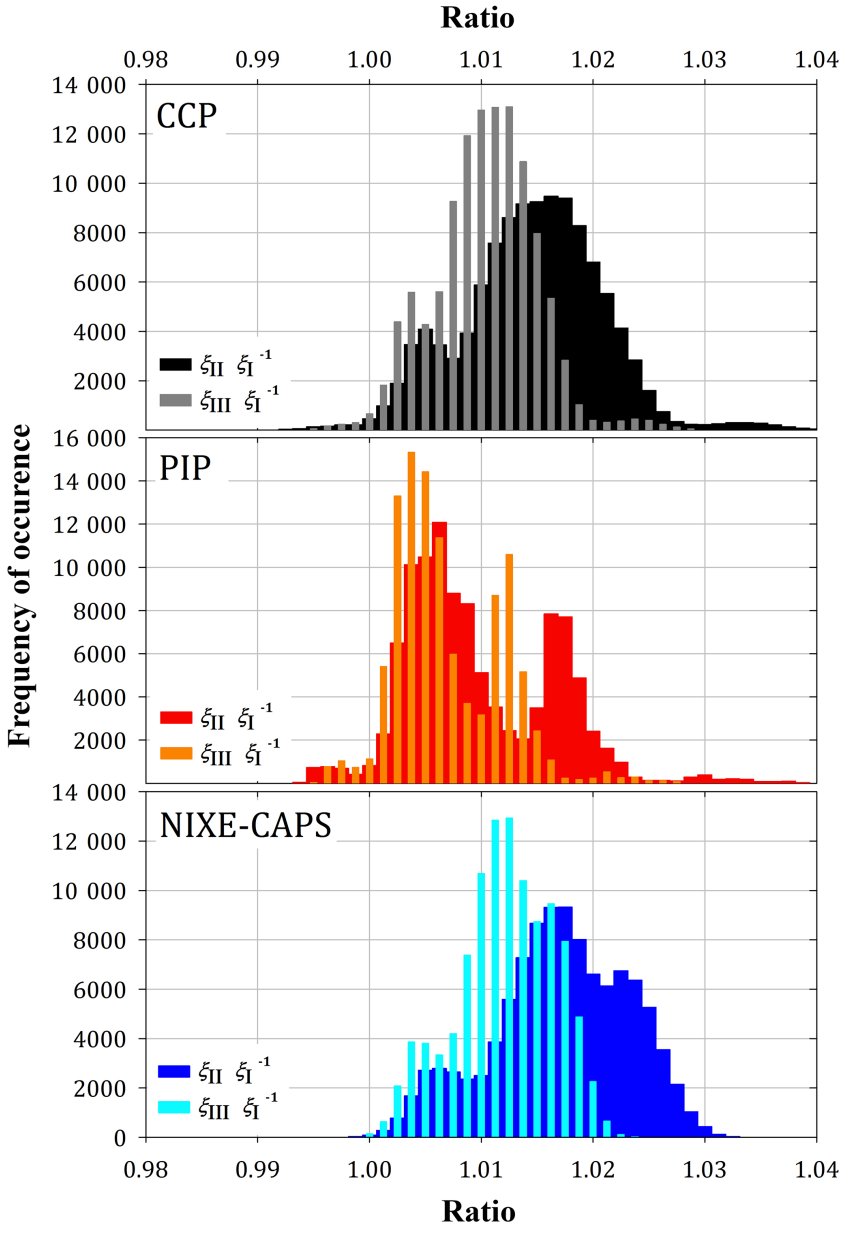

Figure A4. The occurrence frequency of values of $\frac{\xi_{\text {II }}}{\xi_{I}}$ and $\frac{\xi_{I I I}}{\xi_{I}}$ in the data set displayed in Fig. A3.

is used for the following discussions (Sect. 3 and also here in the following).

For the following considerations concerning the congestion-induced compression of a gas volume occurring upstream of a flow obstacle, the resulting increase of the gas' density is described by the factor

$\xi=\frac{\rho_{1}}{\rho_{2}}=\frac{p_{1}}{p_{2}} \frac{T_{2}}{T_{1}}$

by assuming the air to behave as an ideal gas, for which $p$. $V=M \cdot R_{\mathrm{S}} \cdot T$, or rather $p=\rho \cdot R_{\mathrm{S}} \cdot T$, with the static pressure $[p]=\mathrm{kg} \mathrm{m} \mathrm{s}^{-2}$, the gas volume $[V]=\mathrm{m}^{3}$, the mass of the gas $[M]=\mathrm{kg}$, the gas temperature $[T]=\mathrm{K}$, the gas density $[\rho]=\mathrm{kg} \mathrm{m}^{-3}$ and $\mathbf{R}_{\mathrm{s}}$, the specific gas constant in units of $\mathrm{J} \mathrm{kg}^{-1} \mathrm{~K}^{-1}$; while $\mathrm{J}=\mathrm{kg} \mathrm{m}^{2} \mathrm{~s}^{-2}$.

The ambient conditions, at the data boom, are described by $p_{1}, T_{1}$ and the TAS $=v_{1}$ in units of $\mathrm{m} \mathrm{s}^{-1}$.

The measurement conditions, in the detection region upstream of the underwing probe, are described by $p_{2}$ and the $\mathrm{PAS}=v_{2}\left(\right.$ in $\left.\mathrm{m} \mathrm{s}^{-1}\right)$, recorded data from the individual underwing cloud probes CCP, PIP and NIXE-CAPS. 
The consequences of the air compression are $p_{1} \leq p_{2}$ and $T_{1} \leq T_{2}$.

\section{A1 Deriving $\xi$ comprising most of the observational data}

Here we assume that

- the gas properties are those of an ideal gas,

- the principle theorem of thermodynamics - the conservation of energy - is fulfilled,

- the gas flow conditions follow Bernoulli's law.

This allows for expressing

$\frac{1}{2} v_{1}^{2}+h_{1}+\Phi_{1}=\frac{1}{2} v_{2}^{2}+h_{2}+\Phi_{2}$.

With the specific enthalpy $[h]=\mathrm{J} \mathrm{kg}^{-1}$ and provided that following assumption for the gravitational potential is valid $\Phi_{1} \approx \Phi_{2}$, then the air temperature in the instruments measurement region is determined by

$T_{2}=T_{1}+\frac{v_{1}^{2}-v_{2}^{2}}{2 \cdot c_{\mathrm{p}}}$

from which the first expression for the $\xi$ correction follows

$\xi_{\mathrm{I}}=\frac{p_{1}}{p_{2}} \frac{T_{2}}{T_{1}}=\frac{p_{1}}{p_{2}}\left(1+\frac{v_{1}^{2}-v_{2}^{2}}{2 \cdot c_{\mathrm{p}} \cdot T_{1}}\right)$.

\section{A2 Deriving $\xi$ by presuming an adiabatic change of pressure conditions}

Exclusively relying on the measurements of the flow velocity in the detection region and by assuming an adiabatic change of the pressure conditions due to compression an additional assumption is added to those specified in Sect. A1:

$\frac{T_{2}}{T_{1}}=\left(\frac{p_{2}}{p_{1}}\right)^{\kappa} \Leftrightarrow\left(\frac{T_{2}}{T_{1}}\right)^{-\frac{1}{\kappa}}=\frac{p_{1}}{p_{2}}$,

with the adiabatic exponent $\kappa=\frac{R_{\mathrm{s}}}{c_{\mathrm{p}}}$.

The temperature $T_{2}$ is calculated analogously to Eq. (A3). Thus, for the $\xi$ correction, a new expression follows:

$$
\begin{aligned}
\xi_{\text {II }} & =\frac{p_{1}}{p_{2}} \frac{T_{2}}{T_{1}}=\left(\frac{T_{2}}{T_{1}}\right)^{-\frac{1}{\kappa}} \frac{T_{2}}{T_{1}}=\left(\frac{T_{2}}{T_{1}}\right)^{\frac{\kappa-1}{\kappa}} \\
& =\left(1+\frac{v_{1}^{2}-v_{2}^{2}}{2 \cdot c_{\mathrm{p}} \cdot T_{1}}\right)^{\frac{\kappa-1}{\kappa}} .
\end{aligned}
$$

This approach bears a certain inconsistency: the compression-induced increase of the air temperature in the measurement region $\left(T_{2}\right)$ may also comprise diabatic processes, whereas for the compression-induced increase of the static pressure exclusively adiabatic changes of state are assumed to occur.

\section{A3 Deriving $\xi$ by presuming all changes of state as adiabatic}

Relying only on the static pressure individually measured by respective instrument, i.e. by the underwing probes and the data boom, a further assumption to those specified in Appendix A1 is introduced: all changes of state are exclusively adiabatic, i.e. they proceed without any energy exchange with the environment.

Using Eq. (A10) the temperature $T_{2}$ is calculated as

$T_{2}=T_{1} \cdot\left(\frac{p_{2}}{p_{1}}\right)^{\kappa}$.

This allows for expressing the $\xi$ correction as follows:

$\xi_{\mathrm{III}}=\frac{p_{1}}{p_{2}} \frac{T_{2}}{T_{1}}=\frac{p_{1}}{p_{2}}\left(\frac{p_{1}}{p_{2}}\right)^{-\kappa}=\left(\frac{p_{1}}{p_{2}}\right)^{1-\kappa}$.

Note that now the measured air flow velocities are entirely excluded from consideration. The correction with $\xi_{\text {III }}$ relies only on the static air pressures measured by the data boom $\left(p_{1}\right)$ and in the detection region of the underwing probes $\left(p_{2}\right)$. Indeed, the pressure data may be comparably accurate, even when obtained at high flight velocities, and thus measured pressures exhibit certain robustness. Nevertheless, it appears plausible that the compression strength depends on the absolute air flow velocities; however, $\xi_{\text {III }}$ does not anymore account for the values of $v_{1}$ and $v_{2}$.

\section{A4 Sensitivity tests}

In the following the differently derived $\xi$ corrections are evaluated concerning their effectiveness and, by applying them to measurement data from the ML-CIRRUS mission, concerning the comparability of the individual results. The first appraisal aims at the question of whether the assumption of adiabatic changes of state can be confirmed by the in-flight air temperature measurements. Subsequently the agreement of the differently derived $\xi$ corrections $\left(\xi_{\mathrm{I}}, \xi_{\mathrm{II}}, \xi_{\mathrm{III}}\right)$ is proven.

1. The temperature $T_{2}$ obtained according to Eqs. (A2) and (A12) is compared for each of the individual instruments. Therefore, we introduce

$T_{2}^{a}=T_{1}\left(\frac{p_{2}}{p_{1}}\right)^{\kappa} ; T_{2}^{b}=T_{1}+\frac{v_{1}^{2}-v_{2}^{2}}{2 \cdot c_{\mathrm{p}}}$.

In the expression concerning the change of enthalpy $\mathrm{d} h=$ $\mathrm{d} q+\alpha \mathrm{d} p$, with $\alpha=\frac{V}{M}$, the assumption of adiabatic change of state is implied by setting $\mathrm{d} q=0$, i.e. that exchange of heat between the system and the environment is excluded, in accordance to the principle theorem of thermodynamics. This assumption is likely valid as the processes occur very rapidly. When considering that diabatic processes occur, as for example due to mixing or friction, this means that $\mathrm{d} q \neq 0$ and consequently $T_{2}^{a} \neq T_{2}^{b}$. 
With the unprovable assumption that $T_{2}^{b}$ constitutes the reference, for which potentially involved diabatic processes remain considered; the ratio $\frac{T_{2}^{a}}{T_{2}^{b}}$ would indicate the strength of deviation from this insinuated reference. The ratio $\frac{T_{2}^{a}}{T_{2}^{b}}$ is shown as a function of flight velocity in Fig. A1, the occurrence frequency of $\frac{T_{2}^{a}}{T_{2}^{b}}$ values in the data set is displayed in Fig. A2. For all instruments' data the ratio $\frac{T_{2}^{a}}{T_{2}^{b}}$ remains between 0.988 and 1.004, whereas the majority of data ranges between 0.99 and 1.0. As a consequence, the assumption of adiabatic changes of state accompanied with the compression appears justified. The deviation of the ratio $\frac{T_{2}^{a}}{T_{2}^{b}}$ from the adiabatic threshold (black line in Fig. A1) increases with the TAS, as can be expected because the occurrence of turbulence and accompanied mixing with increasing TAS is very conceivable.

In essence, the increasing air temperature upstream of the underwing probes is accompanied with an almost ideal adiabatic compression at flight velocities of up to $250 \mathrm{~m} \mathrm{~s}^{-1}$.

1. The differently derived $\xi$ corrections $\left(\xi_{\mathrm{I}}, \xi_{\mathrm{II}}, \xi_{\mathrm{III}}\right)$ are compared, provided that - once more as an unprovable assumption $-\xi_{\text {I }}$ constitutes the reference. As $\xi_{\text {I mostly }}$ involves observational data, $\xi_{\text {I }}$ is largely independent of assumptions (e.g. the occurrence of exclusively adiabatic processes) that may implicate constraints on the interpretation of the results. In the following the ratios $\frac{\xi_{\text {II }}}{\xi_{\mathrm{I}}}$ and $\frac{\xi_{\text {III }}}{\xi_{\mathrm{I}}}$ are analysed from the individual underwing probes, i.e. CCP, PIP and NIXE-CAPS, respectively. In Fig. A3 the obtained ratios are displayed and in Fig. A4 the corresponding occurrence frequencies. The resulting $\xi$ ratios always remain between 0.985 and 1.045 which indicates a strong agreement between the differently derived $\xi$ that generally exhibit an absolute deviation of less than $5 \%$, in most cases of even less than $2 \%$. Furthermore, the factors $\xi_{\text {I }}$ and $\xi_{\text {III }}$ exhibit the best agreement, regardless of the instrument for which the $\xi$ values are determined. This means that the approach which assumes exclusively adiabatic processes to occur provides the most consistent reproduction of $\xi_{\mathrm{I}}$, whereas $\xi_{\text {I }}$ at the most involves the available observational data.

\section{A5 Conclusions}

From the previous investigation the following conclusions arise:

1. The compression of air occurring upstream of the underwing probes at flight velocities of up to $250 \mathrm{~m} \mathrm{~s}^{-1}$ appears to be described consistently by an ideal adiabatic change of state.

2. The differently derived $\xi$ corrections, i.e. $\xi_{\text {I }}$ (based on observational data) and $\xi_{\text {III }}$ (assuming exclusively adi- abatic changes of state accompanied with the compression), show the best agreement while the correction with $\xi_{\text {II }}$ (comprising exclusively the change of pressure state as adiabatic) leads to the largest deviation from what is expected for an adiabatic process.

3. For those underwing probes lacking an air flow velocity sensor the implementation of a static air pressure sensor of appropriate accuracy may provide a crucial improvement for obtaining a measure of the compression strength at any given flight speed.

\section{Appendix B: Automated spheroid recognition from OAP images}

Distortions of particle images are caused, for example, by false measurements of the speed of particles when they pass the OAP detector region. In terms of shape any liquid cloud particle (with $D_{\mathrm{p}}<2 \mathrm{~mm}$ ) may provide the reference for nearly perfect spherical bodies (Pruppacher and Klett, 2012; Thurai et al., 2009) that are detected with an OAP. Thus, the recorded images of such liquid (spheroidal) cloud elements occasionally could exhibit significant distortions from spherical shape due to strong deviations between the measured and the true particle speed. In such a case the aspect ratio of the particle image deviates from unity and provides a measure for the error of measured particle speed. Provided that a spherical particle produces identical 2-D shadow images, independent from the particle's rotation, the deviation of the image's aspect ratio from unity is individually measurable for each particle image. To identify spheroids in the recorded data, or rather to identify images that presumably emanate from spherical cloud elements, an automatic procedure is used to systematically categorise recorded particle images. Insinuating that the particles' images always exhibit a certain degree of distortion, most of the images should depict an ellipsoidal shape even though the initial cloud particle was potentially spherical.

Of course the categorisation of an imaged cloud particle as spherical cannot be exclusively based on an image's ellipsoidal character. Further criteria are applied by limiting the data set to be analysed to a certain range of ambient air temperatures (e.g. $>270 \mathrm{~K}$ ) or pressure altitudes $(>300 \mathrm{hPa})$ with the purpose of largely excluding ice particles from the analysis, as these would likely impact and falsify the particle categorisation.

However, one of the most important limitations for an automated categorisation of particle images is the available pixel resolution of the recorded images (which is $15 \mu \mathrm{m}$ for the CIPgs-type instruments and is $100 \mu \mathrm{m}$ for the PIP-type OAPs, respectively; cf. Sect. 3.1). From a cloud particle with $D_{\mathrm{p}}<30 \mu \mathrm{m}$, for example, the measurement information from the CIPgs is provided as a $2 \times 2$ pixel image. Due to this low image resolution it is impossible to further distinguish fine microphysical structures, e.g. if the particle surface is 


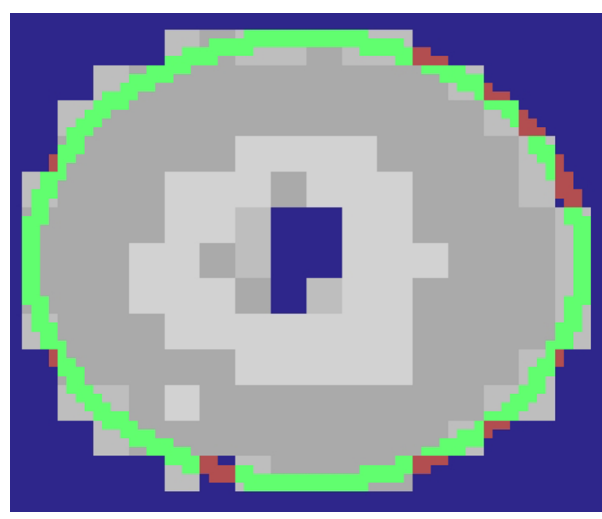

Figure B1. Ellipsoid particle in comparison to the fitted ellipse by using the Bresenham algorithm. The green ellipse pixels denote events when the approximated ellipse hits the particle image pixels (grey shaded). Red ellipse pixels illustrate when particle image pixels are missed by the approximation. The shade brightness of the particle image pixels (grey) illustrates the different grades of shadowing generated by the particles on the OAP diode array detector. The image background (lacking any particle image information) is given in blue.

smoothly rounded (indicative for liquid particles) or rather rough (ice particles). Thus, for the further analysis only those particle images were selected which consist of at least 5 pixels $(75 \mu \mathrm{m})$ in either direction along the image's main axes, i.e. the axis $a$ (in line with the flight direction and perpendicular to the OAP diode array) and the axis $b$ (in line with the OAP diode array).

One further effect that needs to be considered is the consequence of Fresnel diffraction on liquid spherical particles passing the beam of the coherent detection laser. This effect results in particle images exhibiting a bright spot in the centre, referred to as the Poisson spot (Korolev, 2007). The size of the Poisson spot depends on the distance $\left(Z_{\mathrm{d}}\right)$ of the particle from the object plane when passing the detection laser. With increasing distance of a detected particle from the object plane the resulting image is expanded in size and the image's edges get smoothed compared to those of the original particle. Therefore, those images having a Poisson spot resulting from $Z_{\mathrm{d}}>4$ (cf. Korolev, 2007) are discarded from further consideration. Furthermore, images exhibiting a noncontinuous image edge, which appear as open circles or cshapes around a Poisson spot, are also discarded. The approach of Korolev (2007) is used to retrieve the initial size of the airborne cloud particle from the images having a Poisson spot and that remain after previous selection.

To identify the ellipsoidal particle images in the remaining data set, the Bresenham algorithm (Bresenham, 1965) is used to get the shape of particle image approximated by an ellipse (cf. Fig. B1), bearing in mind that a circle is a type of ellipse. The radii of the ellipse are iteratively fitted to the length $(a)$ and the width $(b)$ of the particle image. The comparison of the approximated ellipse with the particle image allows for categorising the particle images. If the imaged particle is too small the available image resolution (cf. above) impedes the approximation of the particle image as an ellipse. Therefore, the resolution of the grid-plane for the ellipse approximation needs to be increased (e.g. by a factor of 4) in comparison to the pixel resolution of images taken. Thus, each image pixel $(15 \mu \mathrm{m} \times 15 \mu \mathrm{m})$ is subdivided by a $4 \times 4$ sub-grid for the ellipse approximation such that an ellipse can be moved and expanded/contracted on a grid of $3.75 \mu \mathrm{m}$ mesh width when projected onto the particle image plane. The implementation of a higher resolved sub-grid has the following advantages:

1. The algorithm aligns the centre of the ellipse to the centre of the particle image with improved accuracy in both cases, i.e. whether the number of image pixels along the main axes is even or uneven.

2. The algorithm enables the adaption of the ellipse's shape to the particle image by expanding or contracting the ellipse's axes on a finer scale to resolve the following optimisation problem.

The optimisation problem is to approximate the ellipse that provides the maximum of conformities and the minimum of nonconformities with the shape of the particle image. In Fig. B1 a result of the automated approximation process is illustrated. Fractions of the approximated ellipse that cause nonconformities are coloured in red, while all conformities between the approximated ellipse and the particle images are depicted in green. The number of conformities $\eta_{\text {conf. over }}$ the entire ellipse $\eta_{\text {total }}$ provides a criterion to automatically discard those images from further consideration that significantly distinguish from an ellipsoidal shape (e.g. irregular shapes, false images). The threshold for this selection criterion was conservatively set to an acceptance value of $\frac{\eta_{\text {conf. }}}{\eta_{\text {total }}} \geq 0.9$ indicating an ellipse as appropriate approximation to the particle image. The ratio of the main axes' lengths $\left(\frac{a}{b}\right)$ of accepted ellipses is assumed to represent the aspect ratio of the selected individual particle image. Of course, some uncertainties are inherent in this approach. The largest ambiguity arises from the optical resolution of the images. Consequently, the remaining uncertainty of the obtained object size in either axis direction is estimated with $\pm 7.5 \mu \mathrm{m}$. The uncertainty of the aspect ratio determined for the individual particle images is obtained as the largest possible error that arises from the ratio $\frac{a+7.5 \mu \mathrm{m}}{b-7.5 \mu \mathrm{m}}$.

With the applied criteria, the computational spheroid recognition may provide an appropriate tool to specifically select OAP image data which emanate with highest probability from the detection of spherical particles that are most likely liquid cloud droplets:

1. by limiting the analysed data set to a certain range of ambient air temperatures and pressure altitudes; 
2. by the automated discarding of false images or images that very obviously emanate from particles of irregular (non-spherical) shape;

3. by the automated rejection of images that emanate from particles passing the detection region too far away from the object plane, which would certainly disturb any conclusion concerning the shape of detected particle;

4. and by the automated approximation of an ellipse to the particle image with severe conformity criteria. 
Acknowledgements. The authors thank the coordinators of the involved missions. We gratefully acknowledge the technical support provided by C. von Glahn, K.-D. Wilhelm, W. Schneider and T. Böttger. We thank M. Schnaiter, E. Järvinen, M. Hermann, K. Kandler, and M. Baumgartner for the fruitful discussions as well as the ML-CIRRUS team for the suggestions (ML-CIRRUS workshop, 25/26 November 2015). Furthermore, the help and advice of R. E. Jubb is highly appreciated as well as the very valuable and constructive suggestions of D. Baumgardner, A. Korolev, C. H. Twohy and of an anonymous referee. This work is based on data acquired during the DFG-supported (SPP1294) HALO missions ML-CIRRUS and ACRIDICON-CHUVA, and this study was prepared with support by the German "Bundesministerium für Bildung und Forschung" (BMBF) within the joint ROMIC project SPITFIRE (01LG1205A). The AIRTOSS-ICE campaign was supported by DFG through the projects BO1829/7-1 and SP1163/31. Our research received funding from the European Research Council under the European Union's Seventh Framework Program (FP/2007-2013)/ERC grant agreement no. 321040 (EXCATRO). Financial support from the Max Planck Institute for Chemistry and the Helmholtz Society is gratefully acknowledged. We particularly thank all aircraft crew members and operators of the research aircraft $H A L O$ and the GFD Learjet-35A for their engagement.

Edited by: D. Baumgardner

Reviewed by: A. Korolev, C. H. Twohy

and one anonymous referee

\section{References}

Abdelmonem, A., Järvinen, E., Duft, D., Hirst, E., Vogt, S., Leisner, T., and Schnaiter, M.: PHIPS-HALO: the airborne Particle Habit Imaging and Polar Scattering probe - Part 1: Design and operation, Atmos. Meas. Tech., 9, 3131-3144, doi:10.5194/amt9-3131-2016, 2016.

Baumgardner, D., Brenguier, J. L., Bucholtz, A., Coe, H., DeMott, P., Garrett, T. J., Gayet, J. F., Hermann, M., Heymsfield, A., Korolev, A., Krämer, M., Petzold, A., Strapp, W., Pilewskie, P., Taylor, J., Twohy, C., Wendisch, M., Bachalo, W., and Chuang, P.: Airborne instruments to measure atmospheric aerosol particles, clouds and radiation: A cook's tour of mature and emerging technology, Atmos. Res., 102, 10-29, 2011.

Baumgardner, D., Jonsson, H., Dawson, W., O'Connor, D., and Newton, R.: The cloud, aerosol and precipitation spectrometer: a new instrument for cloud investigations, Atmos. Res., 59-60, 251-264, 2001.

Baumgardner, D., Newton, R., Krämer, M., Meyer, J., Beyer, A., Wendisch, M., and Vochezer, P.: The Cloud Particle Spectrometer with Polarization Detection (CPSPD): A next generation open-path cloud probe for distinguishing liquid cloud droplets from ice crystals, Atmos. Res., 142, 2-14, 2014.

Baumgardner, D., Strapp, W., and Dye, J. E.: Evaluation of the Forward Scattering Spectrometer Probe. Part II: Corrections for Coincidence and Dead-Time Losses, J. Atmos. Ocean. Tech., 2, 626-632, 1985.

Bresenham, J. E.: Algorithm for computer control of a digital plotter, IBM Syst. J., 4, 25-30, 1965.
Dixon, J. C.: Appendix B: Properties of Air, in: The Shock Absorber Handbook, 361-374, John Wiley \& Sons Ltd, The Atrium, Southern Gate, Chichester, West Sussex, England, 2007.

DMT: Droplet Measurement Technologies Inc., Data Analysis User's Guide, Chapter II: Single Particle Imaging, DOC-0223, 2009.

Drummond, A. M. and MacPherson, J. I.: Aircraft Flow Effects on Cloud Drop Images and Concentrations Measured by the NAE Twin Otter, J. Atmos. Ocean. Tech., 2, 633-643, 1985.

Dye, J. E. and Baumgardner, D.: Evaluation of the Forward Scattering Spectrometer Probe. Part I: Electronic and Optical Studies, J Atmos. Ocean. Tech., 1, 329-344, 1984.

Finger, F., Werner, F., Klingebiel, M., Ehrlich, A., Jäkel, E., Voigt, M., Borrmann, S., Spichtinger, P., and Wendisch, M.: Spectral optical layer properties of cirrus from collocated airborne measurements and simulations, Atmos. Chem. Phys., 16, 7681-7693, doi:10.5194/acp-16-7681-2016, 2016.

Frey, W., Eichler, H., de Reus, M., Maser, R., Wendisch, M., and Borrmann, S.: A new airborne tandem platform for collocated measurements of microphysical cloud and radiation properties, Atmos. Meas. Tech., 2, 147-158, doi:10.5194/amt-2-147-2009, 2009.

Frey, W., Borrmann, S., Kunkel, D., Weigel, R., de Reus, M., Schlager, H., Roiger, A., Voigt, C., Hoor, P., Curtius, J., Krämer, M., Schiller, C., Volk, C. M., Homan, C. D., Fierli, F., Di Donfrancesco, G., Ulanovsky, A., Ravegnani, F., Sitnikov, N. M., Viciani, S., D’Amato, F., Shur, G. N., Belyaev, G. V., Law, K. S., and Cairo, F.: In situ measurements of tropical cloud properties in the West African Monsoon: upper tropospheric ice clouds, Mesoscale Convective System outflow, and subvisual cirrus, Atmos. Chem. Phys., 11, 5569-5590, doi:10.5194/acp-11-55692011, 2011.

Hinds, W. C.: Aerosol Technology: Properties, Behavior, and Measurement of airborne Particles, 2nd edition, 5-21, John Wiley \& Sons Inc., Hoboken, New Jersey, 1999.

Kärcher, B. and Lohmann, U.: A parameterization of cirrus cloud formation: Homogeneous freezing of supercooled aerosols, J. Geophys. Res.-Atmos., 107, 4010, doi:10.1029/2001JD000470, 2002.

King, W. D.: Air Flow and Particle Trajectories around Aircraft Fuselages. I: Theory, J. Atmos. Ocean. Tech., 1, 5-13, 1984.

King, W. D.: Air Flow and Particle Trajectories around Aircraft Fuselages. IV: Orientation of Ice Crystals, J. Atmos. Ocean. Tech., 3, 433-439, 1986a.

King, W. D.: Air Flow around PMS Canisters, J. Atmos. Ocean. Tech., 3, 197-198, 1986b.

Klingebiel, M., de Lozar, A., Molleker, S., Weigel, R., Roth, A. Schmidt, L., Meyer, J., Ehrlich, A., Neuber, R., Wendisch, M., and Borrmann, S.: Arctic low-level boundary layer clouds: in situ measurements and simulations of mono- and bimodal supercooled droplet size distributions at the top layer of liquid phase clouds, Atmos. Chem. Phys., 15, 617-631, doi:10.5194/acp-15617-2015, 2015.

Knollenberg, R. G.: The Optical Array: An Alternative to Scattering or Extinction for Airborne Particle Size Determination, J. Appl. Meteorol., 9, 86-103, 1970.

Korolev, A.: Reconstruction of the Sizes of Spherical Particles from Their Shadow Images. Part I: Theoretical Considerations, J. Atmos. Ocean. Tech., 24, 376-389, 2007. 
Korolev, A., Emery, E., and Creelman, K.: Modification and tests of particle probe tips to mitigate effects of ice shattering, J. Atmos. Ocean. Tech., 30, 690-708, 2013.

Korolev, A., Makarov, Y. E., and Novikov, V.: On the calibration of photoelectric cloud droplet spectrometer FSSP-100, TCAO, 158, 43-49, 1985.

Korolev, A. V., Kuznetsov, S. V., Makarov, Y. E., and Novikov, V. S.: Evaluation of Measurements of Particle Size and Sample Area from Optical Array Probes, J. Atmos. Ocean. Tech., 8, 514-522, 1991.

Korolev, A. V., Strapp, J. W., and Isaac, G. A.: Evaluation of the Accuracy of PMS Optical Array Probes, J. Atmos. Ocean. Tech., 15, 708-720, 1998.

Krämer, M., Schiller, C., Afchine, A., Bauer, R., Gensch, I., Mangold, A., Schlicht, S., Spelten, N., Sitnikov, N., Borrmann, S., de Reus, M., and Spichtinger, P.: Ice supersaturations and cirrus cloud crystal numbers, Atmos. Chem. Phys., 9, 3505-3522, doi:10.5194/acp-9-3505-2009, 2009.

Kulkarni, P., Baron, P. A., and Willeke, K.: Aerosol measurement: principles, techniques, and applications, John Wiley \& Sons, 2571, 2011.

Lance, S., Brock, C. A., Rogers, D., and Gordon, J. A.: Water droplet calibration of the Cloud Droplet Probe (CDP) and inflight performance in liquid, ice and mixed-phase clouds during ARCPAC, Atmos. Meas. Tech., 3, 1683-1706, doi:10.5194/amt3-1683-2010, 2010.

Laursen, K. K., Jorgensen, D. P., Brasseur, G. P., Ustin, S. L., and Huning, J. R.: HIAPER: The Next Generation NSF/NCAR Research Aircraft, B. Am. Meteor. Soc., 87, 896-909, 2006.

Lawson, R. P., O’Connor, D., Zmarzly, P., Weaver, K., Baker, B., Mo, Q., and Jonsson, H.: The 2D-S (Stereo) Probe: Design and Preliminary Tests of a New Airborne, High-Speed, HighResolution Particle Imaging Probe, J. Atmos. Ocean. Tech., 23, 1462-1477, 2006.

MacPherson, J. I. and Baumgardner, D.: Airflow about King Air Wingtip-Mounted Cloud Particle Measurement Probes, J. Atmos. Ocean. Tech., 5, 259-273, 1988.

Menter, F., Kuntz, M., and Langtry, R.: Ten years of industrial experience with the SST turbulence model, Turbul., Heat Mass Transfer, 4, 625-632, 2003.

Meyer, J.: Ice Crystal Measurements with the New Particle Spectrometer NIXE-CAPS, Schriften des Forschungszentrum Jülich, Energy \& Environment, 160, ISBN:978-3-89336-840-2, Forschungszentrum Jülich GmbH, 2012.

Molleker, S., Borrmann, S., Schlager, H., Luo, B., Frey, W., Klingebiel, M., Weigel, R., Ebert, M., Mitev, V., Matthey, R., Woiwode, W., Oelhaf, H., Dörnbrack, A., Stratmann, G., Grooß, J. U., Günther, G., Vogel, B., Müller, R., Krämer, M., Meyer, J., and Cairo, F.: Microphysical properties of synoptic-scale polar stratospheric clouds: in situ measurements of unexpectedly large $\mathrm{HNO}_{3}$ containing particles in the Arctic vortex, Atmos. Chem. Phys., 14, 10785-10801, doi:10.5194/acp-14-10785-2014, 2014.
Naumann, Z. and Schiller, L.: A drag coefficient correlation, Z. Ver. Deutsch Ing., 77, 318-323, 1935.

Norment, H. and Quealy, A.: Three-dimensional trajectory analyses of two drop sizing instruments: PMS OAP and PMS FSSP, AIAA 25th Aerospace Sciences Meeting, 12-15 January 1887, Reno, Nevada, 1988.

Norment, H. G.: Three-Dimensional Trajectory Analysis of Two Drop Sizing instruments: PMS* OAP and PMS* FSSP, J. Atmos. Ocean. Tech., 5, 743-756, 1988.

Pruppacher, H. R. and Klett, J. D.: Microphysics of Clouds and Precipitation, p. 397 and p. 551, DOI:10.1007/978-0-306-48100-0, Springer Netherlands, 2012.

Spichtinger, P. and Gierens, K. M.: Modelling of cirrus clouds Part 1a: Model description and validation, Atmos. Chem. Phys., 9, 685-706, doi:10.5194/acp-9-685-2009, 2009.

Thurai, M., Bringi, V. N., Szakáll, M., Mitra, S. K., Beard, K. V., and Borrmann, S.: Drop Shapes and Axis Ratio Distributions: Comparison between 2D Video Disdrometer and Wind-Tunnel Measurements, J. Atmos. Ocean. Tech., 26, 1427-1432, 2009.

Voigt, C., Schumann, U., Minikin, A., Abdelmonem, A., Afchine, A., Borrmann, S., Boettcher, M., Buchholz, B., Bugliaro, L., Costa, A., Curtius, J., Dollner, M., Dörnbrack, A., Dreiling, V., Ebert, V., Ehrlich, A., Fix, A., Forster, L., Frank, F., Fütterer, D., Giez, A., Graf, K., Grooß, J.-U., Groß, S., Heimerl, K., Heinold, B., Hüneke, T., Järvinen, E., Jurkat, T., Kaufmann, S., Kenntner, M., Klingebiel, M., Klimach, T., Kohl, R., Krämer, M., Krisna, T. C., Luebke, A., Mayer, B., Mertes, S., Molleker, S., Petzold, A., Pfeilsticker, K., Port, M., Rapp, M., Reutter, P., Rolf, C., Rose, D., Sauer, D., Schäfler, A., Schlage, R., Schnaiter, M., Schneider, J., Spelten, N., Spichtinger, P., Stock, P., Walser, A., Weigel, R., Weinzierl, B., Wendisch, M., Werner, F., Wernli, H., Wirth, M., Zahn, A., Ziereis, H., and Zöger, M.: ML-CIRRUS - The airborne experiment on natural cirrus and contrail cirrus with the high-altitude long-range research aircraft HALO, B. Am. Meteor. Soc., doi:10.1175/BAMS-D-15-00213.1, 2016.

Wendisch, M. and Brenguier, J.-L.: Airborne measurements for environmental research: methods and instruments, John Wiley \& Sons, 2013.

Willeke, K. and Baron, P. A.: Aerosol measurement, Principles, techniques and applications, 3-36, Van Nostrand Reinhold: New York, NY, 1993. 